\title{
Chapter 1 \\ Systems of Differential Algebraic Equations in Computational Electromagnetics
}

\author{
Idoia Cortes Garcia, Sebastian Schöps, Herbert De Gersem and Sascha Baumanns
}

\begin{abstract}
Starting from space-discretisation of Maxwell's equations, various classical formulations are proposed for the simulation of electromagnetic fields. They differ in the phenomena considered as well as in the variables chosen for discretisation. This contribution presents a literature survey of the most common approximations and formulations with a focus on their structural properties. The differentialalgebraic character is discussed and quantified by the differential index concept.
\end{abstract}

\subsection{Introduction}

Electromagnetic theory has been established by Maxwell in 1864 and was reformulated into the language of vector calculus by Heavyside in 1891 [63, 50]. A historical overview can be found in the review article [77]. The theory is well understood and rigorously presented in many text books, e.g. [49], [54], [44]. More recently researchers have begun to formulate the equations in terms of exterior calculus and differential forms which expresses the relations more elegantly and metric-free, e.g. [51].

The simulation of three-dimensional spatially distributed electromagnetic phenomena based on Maxwell's equations is roughly 50 years old. An early key contribution was the proposition of the finite difference time domain method (FDTD) by Yee to solve the high-frequency hyperbolic problem on equidistant grids in 1966 [100], and its subsequent generalisations and improvements, e.g. [90]. Among the

Idoia Cortes Garcia, Sebastian Schöps and Herbert De Gersem

Technische Universität Darmstadt, Graduate School of Computational Engineering Dolivostraße 15, 64293 Darmstadt, Germany, e-mail: cortesegsc.tu-darmstadt.de, e-mail: schoepsegsc.tu-darmstadt.de, e-mail: degersemetemf.tu-darmstadt.de

Sascha Baumanns

Universität zu Köln, Mathematisches Institut, Weyertal 86-90, 50931 Köln, Germany, e-mail: sbaumanns@math.uni-koeln.de 
most interesting generalisations are the Finite Integration Technique [97] and the Cell Method [4] because they can be considered as discrete differential forms [21]. Most finite-difference codes formulate the problem in terms of the electric and magnetic field strength and yield ordinary differential equations after space discretisation which are solved explicitly in time. FDTD is very robust and remarkable efficient [67] and is considered to be among the 'top rank of computational tools for engineers and scientists studying electrodynamic phenomena and systems' [91].

Around the same time at which FIT was proposed, circuit simulation programs became popular, e.g. [96, 70] and Albert Ruehli proposed the Partial Element Equivalent Circuit method (PEEC) [79, 80]. PEEC is based on an integral formulation of the equations and utilises Green's functions similarly to the Boundary Element Method (BEM) or the Method of Moments (MOM) as BEM is called in the electromagnetics community [48].

Historically, the Finite Element Method (FEM) was firstly employed to Maxwell's equations using nodal basis functions. For vectorial fields, this produces wrong results known as 'spurious modes' in the literature. Their violation of the underlying structure, or more specifically of the function spaces, is nowadays well understood. Nédélec proposed his edge elements in 1980 [72] which are also known as Whitney elements [17]. A rigorous mathematical discussion can be found in many text books, e.g. [66], [3] and [5]. Albeit less wide spread, the application of nodal elements is still popular, for example in the context of discontinuous Galerkin FEM [53, 45]. Also equivalences among the methods have been shown, most prominently FIT can be interpreted on hexahedral meshes as lowest order Nédélec FEM with mass lumping [21, 14].

From an application point of view, electromagnetic devices may behave very differently, e.g. a transformer in a power plant and an antenna of a mobile phone are both described by the same set of Maxwell's equations but still feature different phenomena. Therefore, engineers often solve subsets (simplifications) of Maxwell's equation that are relevant for their problem, for example the well-known eddycurrent problem [49, 39, 76] or the well-known wave equation [91]. For each, one or more formulations have been proposed. They are either distinguished by the use of different variables or gauging conditions [25, 24].

It follows from the variety of simplifications and formulations that discretisation methods have individual strengths and weaknesses for the different classes of applications. For example, the formulation used in FDTD relies on an explicit time integration method which is particularly efficient if the mass matrices are easily invertible, i.e., if they are diagonal or at least block diagonal [20]. This allows FDTD to solve problems with several billions of degrees of freedom. Classical FEM is less commonly applied in that case but one may also analyse high-frequency electromagnetic phenomena in the frequency domain, either to investigate resonance behaviour [98] or source problems with right-hand-sides that can be assumed to vary sinusoidally at a given frequency. In these cases, one solves smaller complexvalued linear systems but the reduced sparsity due to FEM is counteracted by the flexibility in the mesh generation [13]. Also coupling Maxwell's equations to other 


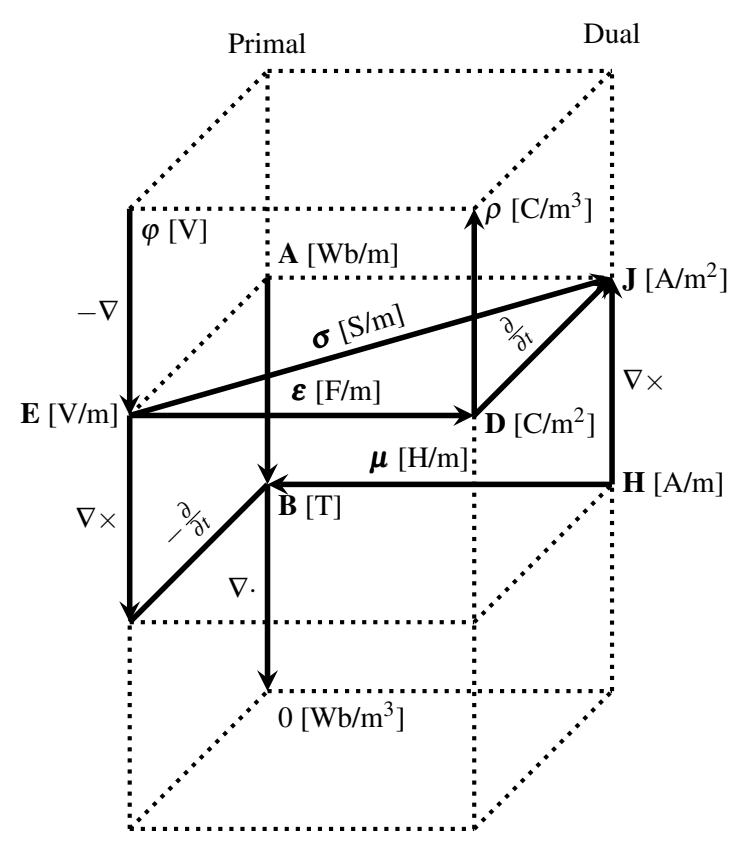

Fig. 1.1 Maxwell's house, based on similar diagrams in [16 38 93]. The concept of duality is for example discussed in the framework of differential forms in [51] and using traditional vector calculus in [54, Section 6.11].

physics may require tailored formulations, see for example for applications in the field of semiconductors [83, 81].

In the low-frequency regime the situation is often more involved since one deals with degenerated versions of Maxwell's equations as certain contributions to the equation vanish in the static limit and the original system becomes unstable [73, 55, 42]. One often turns to approximations of Maxwell's equations as the well-known eddy-current problem. These approximate formulations are often more complicated as they may yield parabolic-semi-elliptic equations that become eventually systems of differential-algebraic equations (DAEs) after space discretisation. The resulting systems are commonly integrated in time domain by fully or linearimplicit methods, e.g. [71, 32]. Only recently, explicit method gained again interest, [6, 87, 40].

Most circuit and electromagnetic field formulations yield DAE systems; the first mathematical treatment of such problems can be traced back to the 60s [60] but gained increased interest in the 80s, e.g. [75]. An important concept in the analysis of DAEs and their well-posedness are the various index concepts, which try to quantify the difficulty of the numerical time-domain solution, see e.g. [75]. This paper discusses the most important low and high-frequency formulations in computational electromagnetics with respect to their differential index. An detailed introduction of 
the index and its variants is not discussed here and the reader is referred to text books and survey articles $[22,47,60,64]$.

This paper summarises relevant discrete formulations stemming from Maxwell's equations. It collects the corresponding known DAE results from the literature, i.e., [71, 94, 7, 9], homogenises their notation and discusses a few missing cases. Each problem is concretised by a mathematical description and specification of an example. The corresponding source code is freely available such that these example can be used as benchmarks, e.g. for the development of time integrators or numerical tools to analyse differential equations.

The paper is organised as follows: Section 1.2 discusses Maxwell's equations, the relevant material relations and boundary conditions. The classical low-frequency approximations and electromagnetic potentials are introduced. Section 1.3 outlines the spatial discretisation in terms of the finite element method and the finite integration technique. After establishing the DAE index concept in Section 1.4, the various discrete formulations are derived. They are discussed separately for the high-frequency full-wave case in Section 1.5 and the quasistatic approximations in Section 1.6. Finally, conclusion are drawn in Section 1.7

\subsection{Maxwell's Equations}

Electromagnetic phenomena are described on the macroscopic level by Maxwell's equations [63, 50, 49, 54, 44] Those can be studied in a standstill frame of reference in integral form

$$
\begin{aligned}
\int_{\partial A} \mathbf{E} \cdot \mathrm{d} \mathbf{s} & =-\int_{A} \frac{\partial \mathbf{B}}{\partial t} \cdot \mathrm{d} \mathbf{A}, \\
\int_{\partial V} \mathbf{D} \cdot \mathrm{d} \mathbf{A} & =\int_{V} \rho \mathrm{d} V, \\
\int_{\partial A} \mathbf{H} \cdot \mathrm{d} \mathbf{s} & =\int_{A}\left(\frac{\partial \mathbf{D}}{\partial t}+\mathbf{J}\right) \cdot \mathrm{d} \mathbf{A}, \\
\int_{\partial V} \mathbf{B} \cdot \mathrm{d} \mathbf{A} & =0,
\end{aligned}
$$

for all areas $A$ and volumes $V \subset \mathbb{R}^{3}$. Using Stokes and Gauß' theorems one derives a set of partial differential equations, see e.g. [5], Chapter 1.1.2] for a mathematical discussion on their equivalence, 


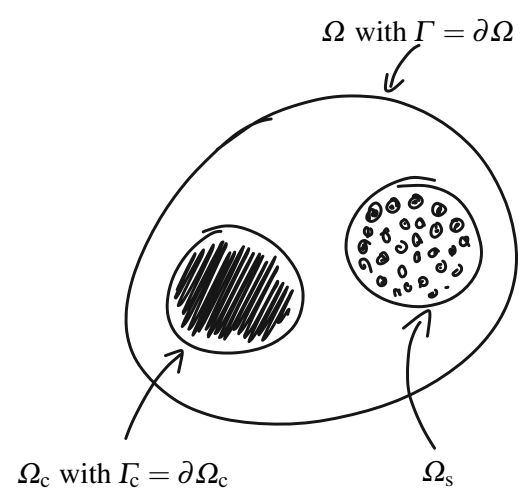

Fig. 1.2 Sketch of domain.

$$
\begin{aligned}
\nabla \times \mathbf{E} & =-\frac{\partial \mathbf{B}}{\partial t}, \\
\nabla \times \mathbf{H} & =\frac{\partial \mathbf{D}}{\partial t}+\mathbf{J}, \\
\nabla \cdot \mathbf{D} & =\rho, \\
\nabla \cdot \mathbf{B} & =0,
\end{aligned}
$$

with $\mathbf{E}$ the electric field strength, $\mathbf{B}$ the magnetic flux density, $\mathbf{H}$ the magnetic field strength, $\mathbf{D}$ the electric flux density and $\mathbf{J}$ the electric current density composed of conductive and source currents, being vector fields $\mathscr{I} \times \Omega \rightarrow \mathbb{R}^{3}$ depending on space $\mathbf{r} \in \Omega$ and time $t \in \mathscr{I}$. The electric charge density $\rho: \mathscr{I} \times \Omega \rightarrow \mathbb{R}$ is the only scalar field. Finally $A$ and $V$ are all areas (respectively volumes) in $\Omega$.

Assumption 1 (Domain) The domain $\Omega \subset \mathbb{R}^{3}$ is open, bounded, Lipschitz and contractible (simply connected with connected boundary, see e.g., [17]).

Maxwell's equations give raise to the so-called de Rham complex, see e.g. [17]. It describes abstractly the relation of the electromagnetic fields in terms of the images and kernels of the differential operators. A simple visualisation is given in Fig. 1.1. This diagram is sometimes called Tonti diagram [93], Deschamps diagram [38], or, in the special case of Maxwell's equations, Maxwell's house [16, 18].

Exploiting the fact that the divergence of a curl vanishes, one can derive from Ampère-Maxwell's law $1.2 \mathrm{~b}-(1.2 \mathrm{c})$ the continuity equation

$$
0=\frac{\partial \rho}{\partial t}+\nabla \cdot \mathbf{J}
$$

which can be interpreted in the static case as Kirchhoff's current law. 


\subsubsection{Boundary Conditions and Material Relations}

To mimic the behaviour of the electromagnetic field of an infinite domain on a truncated computational domain and to model field symmetries, boundary conditions are imposed on $\Gamma=\partial \Omega$. We restrict ourselves to homogeneous electric ('ebc') and magnetic boundary conditions ('mbc')

$$
\begin{cases}\mathbf{n} \times \mathbf{E}=0 & \text { in } \Gamma_{\mathrm{ebc}}, \\ \mathbf{n} \times \mathbf{H}=0 & \text { in } \Gamma_{\mathrm{mbc}},\end{cases}
$$

where $\mathbf{n}$ is the outward normal to the boundary, $\Gamma_{\mathrm{ebc}} \cup \Gamma_{\mathrm{mbc}}=\Gamma$ and $\Gamma_{\mathrm{ebc}} \cap \Gamma_{\mathrm{mbc}}=\emptyset$.

Remark 1. Electrical engineers typically use the physical notation of electric ('ebc') or magnetic ('mbc') boundary conditions rather than the mathematical terminology of 'Dirichlet' or 'Neumann' conditions. The reason is that the mathematical distinction depends on the particular formulation, i.e. the variables chosen to describe the problem, while the physical point of view remains the same. For example in an $E$-based formulation, ebc and mbc correspond to Dirichlet and Neumann conditions, respectively, whereas in an $H$-based formulation, ebc and mbc correspond to Neumann and Dirichlet conditions, respectively.

The fields in Maxwell's equations are further related to each other by the material relations

$$
\mathbf{D}=\boldsymbol{\varepsilon} \mathbf{E}, \quad \quad \mathbf{J}_{\mathrm{c}}=\boldsymbol{\sigma} \mathbf{E}, \quad \mathbf{H}=\boldsymbol{v} \mathbf{B},
$$

where the permittivity $\boldsymbol{\varepsilon}$, conductivity $\boldsymbol{\sigma}$ and reluctivity (inverse permeability $\boldsymbol{\mu}) \boldsymbol{v}$ are rank-2 tensor fields, $\boldsymbol{\xi}: \Omega \rightarrow \mathbb{R}^{3 \times 3}, \boldsymbol{\xi} \in\{\boldsymbol{\varepsilon}, \boldsymbol{\sigma}, \boldsymbol{\mu}, \boldsymbol{v}\}$, whose possible polarisation or magnetisation and nonlinear or hysteretic dependencies on the fields are disregarded in the following for simplicity of notation and $\mathbf{J}_{\mathrm{c}}: \mathscr{I} \times \Omega \rightarrow \mathbb{R}^{3}$ is the conduction current density. With these material relations one defines the total current density as

$$
\mathbf{J}=\mathbf{J}_{\mathrm{c}}+\mathbf{J}_{\mathrm{s}}
$$

where $\mathbf{J}_{\mathrm{S}}$ is a given source current density that represents for example the current density impressed by a stranded conductor [88].

We assume the following material and excitation properties as shown in Fig. 1.2. see also [2, 88] for a more rigorous discussion.

Assumption 2 (Material) The permittivity and permeability tensors, i.e., $\boldsymbol{\varepsilon}$ and $\boldsymbol{\mu}$, are positive definite on the whole domain $\Omega$ and only depend on space $\mathbf{r}$. The conductivity tensor is positive definite on a subdomain $\Omega_{\mathrm{c}} \subset \Omega$ and vanishes elsewhere, i.e., $\operatorname{supp}(\boldsymbol{\sigma})=\Omega_{\mathrm{c}}$. The source current density is defined on the subdomain $\Omega_{\mathrm{s}} \subset \Omega$ with $\Omega_{\mathrm{c}} \cap \Omega_{\mathrm{s}}=\emptyset$, such that $\operatorname{supp}\left(\mathbf{J}_{\mathrm{s}}\right)=\Omega_{\mathrm{s}}$.

This assumption describes the situation of an excitation given by one or several stranded conductors. The key assumption behind this model is a homogeneous cur- 
rent distribution which is justified in many situations, since the individual strands have diameters small than the skin depth and are therefore not affected by eddy currents, i.e. $\Omega_{\mathrm{c}}$ and $\Omega_{\mathrm{S}}$ are disjoint. Other models, e.g. solid and foil conductors, are not covered here. However, it can be shown that the various models can be transformed into each other and thus have similar properties [86].

\subsubsection{Modelling of excitations}

The excitation has been given in (1.6) by the known source current density $\mathbf{J}_{\mathrm{S}}$ which is typically either determined by given voltage drops $u_{k}: \mathscr{I} \rightarrow \mathbb{R}$ or lumped currents $i_{k}: \mathscr{I} \rightarrow \mathbb{R}$. The 3D-0D coupling is governed by so-called conductor models. Besides the solid and stranded models [12], also more elaborated conductors have been proposed, e.g., foil-conductor models [35].

The source current density $\mathbf{J}_{\mathrm{s}}$ is not necessarily solenoidal, i.e.

$$
\nabla \cdot \mathbf{J}_{\mathrm{s}} \neq 0 .
$$

Divergence-freeness is only required for the total current density $\mathbf{J}$ in the absence of charge variations due to the continuity equation (1.3). This has been exploited e.g. in [88, Figure 3] to increase the sparsity of the coupling matrices. However, most conductor models enforce this property such that the source current can be given alternatively in terms of a source magnetic field strength

$$
\mathbf{J}_{\mathrm{s}}=\nabla \times \mathbf{H}_{\mathrm{s}} .
$$

In [88] the abstract framework of winding density functions was proposed. It unifies the individual stranded, solid and foil conductor models and denotes them abstractly by

$$
\chi_{k}: \Omega \rightarrow \mathbb{R}^{3}
$$

with an superscript if needed to distinguish among models, e.g. (i) for stranded and (u) solid conductors. In the simplest case they are characteristic functions with a given orientation.

Example 1. If $\Omega_{\mathrm{s}}=\Omega_{\mathrm{s}, 1} \cup \Omega_{\mathrm{s}, 2} \cup \Omega_{\mathrm{s}, 3}$ consists of two parts of a winding oriented in $z$-direction, each with cross section $A_{k}$ and made of $N_{k}$ strands, and a massive bar with length $\ell_{3}$ aligned with the $z$-direction, the source current is given by

$$
\mathbf{J}_{\mathrm{s}}=\sum_{k=1}^{2} \boldsymbol{\chi}_{k}^{(\mathrm{i})} i_{k}+\boldsymbol{\sigma} \boldsymbol{\chi}_{3}^{(\mathrm{u})} u_{3}
$$

The winding density functions for the stranded conductor model are

$$
\boldsymbol{\chi}_{k}^{(\mathrm{i})}(\mathbf{r})= \begin{cases}\frac{N_{k}}{A_{k}} \mathbf{n}_{z} & \mathbf{r} \in \Omega_{\mathrm{s}, k} \\ 0 & \text { otherwise }\end{cases}
$$


and the unit vector in $z$-direction is denoted by $\mathbf{n}_{z}$. The stranded conductor model distributes an applied current in a homogeneous way such that the individual strands are neither spatially resolved nor modelled as line currents which would cause a too high computational effort. There are many proposals in the literature on how to construct them, most often a Laplace-type problem is solved on the subdomain $\Omega_{s}$, see e.g. [37, 41, 88]. The winding function for the solid conductor is

$$
\chi_{3}^{(\mathrm{u})}(\mathbf{r})= \begin{cases}\frac{1}{\ell_{3}} \mathbf{n}_{z} & \mathbf{r} \in \Omega_{\mathrm{s}, 3} \\ 0 & \text { otherwise }\end{cases}
$$

The solid conductor model homogeneously distributes an applied voltage drop in the massive-conductor's volume.

The winding density functions allow to retrieve global quantities in a postprocessing step, i.e., the current through a solid conductor model is calculated by

$$
i_{k}=\int_{\Omega} \boldsymbol{\chi}_{k}^{(\mathrm{u})} \cdot \mathbf{J} \mathrm{d} V
$$

and the voltage induced along a stranded conductor model follows from

$$
u_{k}=-\int_{\Omega} \boldsymbol{\chi}_{k}^{(\mathrm{i})} \cdot \mathbf{E} \mathrm{d} V .
$$

The expressions (1.8), (1.11) and (1.12) can also be used to set up a field-circuit coupled model [36].

An important property postulated in [88] is that winding functions should fulfil a partition of unity property. The integration of $\boldsymbol{\chi}_{k}(\mathbf{r})$ along a line $\ell_{k}$ between both electrodes of a solid conductor gives always 1 and analogously, $\boldsymbol{\chi}_{k}^{(\mathrm{i})}(\mathbf{r})$ integrated over any cross-sectional plane $A_{k}$ of a stranded conductor should equal the number of turns $N_{k}$ of the winding:

$$
\int_{\ell_{k}} \boldsymbol{\chi}_{k}^{(\mathrm{u})} \cdot \mathrm{d} \mathbf{s}=1, \quad \forall \ell_{k} \quad \text { and } \quad \int_{A_{k}} \boldsymbol{\chi}_{k}^{(\mathrm{i})} \cdot \mathrm{d} \mathbf{S}=N_{k}, \quad \forall A_{k} .
$$

Furthermore, conductor models should not intersect, i.e., [7]

$$
\chi_{i} \cdot \chi_{j} \equiv 0 \quad \text { for } \quad i \neq j
$$

where $\boldsymbol{\chi}_{i}$ and $\boldsymbol{\chi}_{j}$ are winding functions of any type.

For simplicity of notation, we will restrict us in the following to the case of nonintersecting stranded conductors models, i.e.

Assumption 3 (Excitation) The source current density is given by $n_{\text {str }}$ winding functions that fulfil (1.13) and (1.14) such that the excitation is given by

$$
\mathbf{J}_{s}=\sum_{k=1}^{n_{\text {str }}} \boldsymbol{\chi}_{k} i_{k} \quad \text { where } \quad \boldsymbol{\chi}_{k} \equiv \boldsymbol{\chi}_{k}^{(i)} .
$$




\subsubsection{Static and Quasistatic Fields}

Following the common classification of slowly varying electromagnetic fields, [39], we introduce the following definition for quasistatic and static fields

Definition 1. (Simplifications) The fields in Equation (1.2) are called

(a) static if the variation of the magnetic and electric flux densities is disregarded:

$$
\frac{\partial}{\partial t} \mathbf{B}=0 \quad \text { and } \quad \frac{\partial}{\partial t} \mathbf{D}=0
$$

(b) electroquasistatic if the variation of the magnetic flux density is disregarded:

$$
\frac{\partial}{\partial t} \mathbf{B}=0
$$

(c) magnetoquasistatic if the variation of the electric flux density is disregarded:

$$
\frac{\partial}{\partial t} \mathbf{D}=0
$$

(d) full wave if no simplifications are made.

In contrast to the full Maxwell's equations, the classical quasistatic approximations above feature only first order derivatives w.r.t. to time. However, there is another model for slowly varying fields that does not fit into this categorisation, the so-called Darwin approximation, e.g. [61]. It considers the decomposition of the electric field strength $\mathbf{E}=\mathbf{E}_{\text {irr }}+\mathbf{E}_{\text {rem }}$ into an irrotational part $\mathbf{E}_{\text {irr }}$ and a remainder part $\mathbf{E}_{\text {rem. }}$. In contrast to (a) (c) the Darwin approximation only neglects the displacement currents related to $\mathbf{E}_{\text {rem }}$ from the law of Ampère-Maxwell $1.2 \mathrm{~b}$. It still considers second order time derivatives.

The various approximations neglect the influence of several transient phenomena with respect to others, which implicitly categorises fields into primary and secondary ones. For example, let us consider a magnetoquasistatic situation, i.e., the displacement current density $\frac{\partial}{\partial t} \mathbf{D}=0$ is disregarded. This still allows the electric field $\frac{\partial}{\partial t} \mathbf{E} \neq 0$ to vary. However, this variation implies that there is a secondary displacement current density $\frac{\partial}{\partial t} \mathbf{D}=\frac{\partial}{\partial t} \boldsymbol{\varepsilon} \mathbf{E} \neq 0$ which is in the formulation not further considered.

Remark 2. Depending on the application, an electrical engineer chooses the formulation that is best suited for the problem at hand. Typically the physical dimensions, the materials and the occurring frequency are used to estimate which simplification is acceptable, see e.g. [49, 82, 89]. 


\subsubsection{Electromagnetic Potentials}

Typically, one combines the relevant Maxwell equations into a formulation by defining appropriate potentials. One possibility is the $\mathbf{A}-\phi$ formulation [56, 23, 17], where a magnetic vector potential $\mathbf{A}: \mathscr{I} \times \Omega \rightarrow \mathbb{R}^{3}$ and an electric scalar potential $\phi: \mathscr{I} \times \Omega \rightarrow \mathbb{R}$ follow as integration constants from integrating the magnetic Gauss law and Faraday-Lenz' law in space, i.e.,

$$
\mathbf{B}=\nabla \times \mathbf{A} \quad \text { and } \quad \mathbf{E}=-\frac{\partial \mathbf{A}}{\partial t}-\nabla \phi .
$$

The magnetic flux density $\mathbf{B}$ defines the magnetic vector potential A only up to a gradient field. For a unique solution an additional gauging condition is required [23, 62, 31].

A different approach can be taken with the $\mathbf{T}-\Omega$ formulation in case of a magnetoquasistatic approximation (Definition 1.c)] [26, 95, 25]. Here, an electric vector potential $\mathbf{T}: \mathscr{I} \times \Omega \rightarrow \mathbb{R}^{3}$ and a magnetic scalar potential $\psi: \mathscr{I} \times \Omega \rightarrow \mathbb{R}$ describe the fields as

$$
\mathbf{J}_{\mathrm{c}}=\nabla \times \mathbf{T} \quad \text { and } \quad \mathbf{H}=\mathbf{H}_{\mathrm{s}}+\mathbf{T}-\nabla \psi,
$$

with $\nabla \times \mathbf{H}_{\mathrm{s}}=\mathbf{J}_{\mathrm{s}}$. Again, to ensure uniqueness of solution, an additional gauge condition is necessary for $\mathbf{T}$. In contrast to the $\mathbf{A}-\phi$-formulation, the electric vector potential $\mathbf{T}$ is only non-zero on $\Omega_{\mathrm{c}}$.

Existence and uniqueness of the continuous solution will not be discussed in this contributions, see for example [2, 42] for several formulations in the frequency domain case with anisotropic materials and mixed boundary conditions.

The boundary conditions introduced in (1.4) can now be translated into expressions involving only the potentials. This yields for the A- $\phi$-formulation

$$
\begin{cases}\mathbf{n} \times \mathbf{A}=0, \phi=0 & \text { on } \Gamma_{\mathrm{ebc}} \\ \mathbf{n} \times(v \nabla \times \mathbf{A})=0, & \text { on } \Gamma_{\mathrm{mbc}}\end{cases}
$$

and the for the T- $\Omega$ one

$$
\begin{cases}\mu \frac{\partial \psi}{\partial \mathbf{n}}=0, & \text { on } \Gamma_{\mathrm{ebc}} \\ \mathbf{n} \times \nabla \psi=0, & \text { on } \Gamma_{\mathrm{mbc}}\end{cases}
$$

For the electric vector potential $\mathbf{T}$ in the $\mathbf{T}-\Omega$ formulation, boundary conditions have to be set on the corresponding subdomain where it is defined $\Gamma_{\mathrm{c}}=\partial \Omega_{\mathrm{c}}$. This leads to electric boundary conditions

$$
\mathbf{n}_{\mathrm{c}} \times \mathbf{T}=0 \quad \text { on } \Gamma_{\mathrm{c}}
$$

with, analogous to the cases before, $\mathbf{n}_{\mathrm{c}}$ being the outward normal unit vector of $\Gamma_{\mathrm{c}}$. 


\subsection{Spatial Discretisation}

Starting from a differential formulation the Ritz-Galerkin the FE method can be applied using the appropriate Whitney basis functions [66]. Alternatively, FIT or similarly the Cell Method provide a spatial discretisation of Maxwell's equations based on the integral form [97, 4]. In the lowest order case FE and FIT only differ by quadrature, i.e., FIT uses the midpoint rule [14]. We derive in the following the discretisation of the partial differential operators in the terminology of FIT on an hexahedral grid since this allows a simple and explicit construction of divergence, curl and gradient matrices which will aid the following discussion.

\subsubsection{Domain and Grid}

The domain $\Omega$ is decomposed into an oriented simplicial complex that forms the computational grid. For the explanation, it is considered to be a brick and the grid is defined in cartesian coordinates as

$$
\begin{array}{r}
G=\left\{V\left(i_{x}, i_{y}, i_{z}\right) \subset \mathbb{R}^{3} \mid V\left(i_{x}, i_{y}, i_{z}\right)=\left[x_{i_{x}}, x_{i_{x}+1}\right] \times\left[y_{i_{y}}, y_{i_{y}+1}\right] \times\left[z_{i_{z}}, z_{i_{z}+1}\right],\right. \text { for } \\
\left.i_{x}=1, \ldots, n_{x}-1 ; i_{y}=1, \ldots, n_{y}-1 ; i_{z}=1, \ldots, n_{z}-1\right\} .
\end{array}
$$

The elements $V\left(i_{x}, i_{y}, i_{z}\right)=V(n)$ are numbered consecutively with an index $n$ :

$$
n\left(i_{x}, i_{y}, i_{z}\right)=i_{x} k_{x}+\left(i_{y}-1\right) k_{y}+\left(i_{z}-1\right) k_{z}
$$

with $k_{x}=1, k_{y}=n_{x}$ and $k_{z}=n_{x} n_{y}$. Our discrete field quantities can be defined on several geometrical objects such as points $P(n)$, edges $L_{\omega}(n)$ or facets $A_{\omega}(n)$. An edge $L_{\omega}(n)$ connects points $P(n)$ and $P\left(n+k_{\omega}\right)$ in $\omega=\{x, y, z\}$ direction. The facet $A_{\omega}(n)$ is defined by its smallest possible point $P(n)$ and directed such that its normal vector points towards $\omega$. There are $N=n_{x} n_{y} n_{z}$ points and as each point defines three edges and facets, there are in total $N_{\text {dof }}=3 n_{x} n_{y} n_{z}$ edges and facets, ordered in $x, y$ and finally $z$-direction.

Nowadays, inspired by the notation of differential forms, it is well understood Fig. 1.3 Primal and dual grid cells. that a consistent mimetic discretisation of Maxwell's equations requires a primal/dual mesh pair. Even the discretisation with Whitney FEs implicitly constructs a dual mesh [17]. This can be traced back to the inherent structure of Maxwell's equations which are formed with quantities being dual to each other (see [54, Section 6.11]) that are linked by material properties (hodge operators in the terminology of differential forms). This concept is for example rigorously introduced in [51]. 
In contrast to FEM, both FIT and the Cell Method define the second (dual) grid $\tilde{G}$ explicitly. It is obtained by taking the centre of the cells in $G$ as dual grid points (see Figure 1.3). Now the dual quantities can be defined on the dual points $\widetilde{P}(n)$, edges $\widetilde{L}_{\omega}(n)$, facets $\widetilde{A}_{\omega}(n)$ and volumes $\widetilde{V}_{n}$. Dual edges and facets are truncated at the boundary [99].

\subsubsection{Maxwell's Grid Equations}

To illustrate the construction of the operator matrices, Faraday-Lenz's law in integral form, i.e. Equation (1.1a),

$$
\int_{\partial A} \mathbf{E} \cdot \mathrm{d} \mathbf{s}=-\int_{A} \frac{\partial \mathbf{B}}{\partial t} \cdot \mathrm{d} \mathbf{A},
$$

is used as an example. The equality must be fulfilled for all areas $A$, in particular for each facet $A_{\omega}(i, j, k)$ of the computational grid $G$. For the case $\omega=z$,

$$
\widehat{\mathbf{e}}_{x}(i, j, k)+\widehat{\mathbf{e}}_{y}(i+1, j, k)-\widehat{\mathbf{e}}_{x}(i, j+1, k)-\widehat{\mathbf{e}}_{y}(i, j, k)=-\frac{\mathrm{d}}{\mathrm{d} t} \widehat{\mathbf{b}}_{z}(i, j, k),
$$

with

$$
\widehat{\mathbf{e}}_{\omega}(i, j, k)=\int_{L_{\omega}(i, j, k)} \mathbf{E} \cdot \mathrm{d} \mathbf{s} \quad \text { and } \quad \widehat{\mathbf{b}}_{z}(i, j, k)=-\int_{A_{z}(i, j, k)} \mathbf{B} \cdot \mathrm{d} \mathbf{A} .
$$

This procedure is carried out for all the facets of $G$ and the following matrix equation

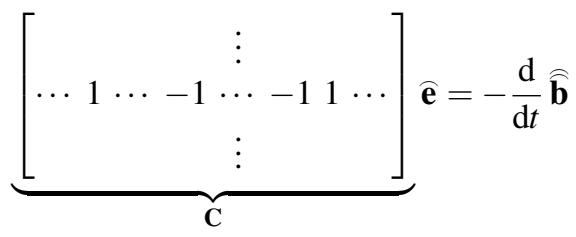

is obtained, which describes Faraday's law in our grid. The matrix $\mathbf{C}$ applies the curl operator on quantities integrated along edges. Similarly, the divergence matrix $\mathbf{S}$, acting on surface integrated degrees of freedom and the gradient matrix $\mathbf{G}$ are built. The same strategy is followed to obtain the matrices for the dual grid $\widetilde{\mathbf{C}}, \widetilde{\mathbf{S}}$ and $\widetilde{\mathbf{G}}$. It can be shown that the matrices mimic all classical identities of vector field on the discrete level, e.g. [85] and [86, Appendix A]. With this, the semi-discrete Maxwell's Grid Equations 


$$
\begin{aligned}
& \mathbf{C} \widehat{\mathbf{e}}=-\frac{\mathrm{d}}{\mathrm{d} t} \widehat{\mathbf{b}}, \\
& \widetilde{\mathbf{C}} \widehat{\mathbf{h}}=\frac{\mathrm{d}}{\mathrm{d} t} \widehat{\mathbf{d}}+\widehat{\mathbf{j}}, \\
& \mathbf{S} \widehat{\mathbf{b}}=0, \\
& \widetilde{\mathbf{S}} \widehat{\mathbf{d}}=\mathbf{q}
\end{aligned}
$$

are obtained which are closely resemble the system 1.2 . The matrices $\mathbf{C}, \widetilde{\mathbf{C}} \in$ $\{-1,0,1\}^{N_{\text {dof }} \times N_{\text {dof }}}$ are the discrete curl operators, $\mathbf{S}, \widetilde{\mathbf{S}} \in\{-1,0,1\}^{N \times N_{\text {dof }}}$ the discrete divergence operators, which are all defined on the primal and dual grid, respectively. The fields are semi-discretely given by $\widehat{\mathbf{e}}, \widehat{\mathbf{h}}, \widehat{\mathbf{d}}, \widehat{\mathbf{j}}, \widehat{\mathbf{b}}: \mathscr{I} \rightarrow \mathbb{R}^{N_{\text {dof }}}$ and $\mathbf{q}: \mathscr{I} \rightarrow \mathbb{R}^{N}$, and correspond to integrals of electric and magnetic voltages, electric fluxes, electric currents, magnetic fluxes and electric charges, respectively.

Lemma 1 The operator matrices fulfil the following properties [99]

- divergence of the curl and curl of the gradient vanish on both grids

$$
\mathbf{S C}=0, \widetilde{\mathbf{S}} \widetilde{\mathbf{C}}=0 \text { and } \mathbf{C G}=0, \widetilde{\mathbf{C}} \widetilde{\mathbf{G}}=0
$$

- primal (dual) gradient and dual (primal) divergence fulfill

$$
\mathbf{G}=-\widetilde{\mathbf{S}}^{\top} \text { and } \quad \widetilde{\mathbf{G}}=-\mathbf{S}^{\top}
$$

- curl and dual curl are related by

$$
\widetilde{\mathbf{C}}=\mathbf{C}^{\top}
$$

Furthermore, potentials can be introduced on the primal grid, i.e.

$$
\widehat{\mathbf{e}}=-\frac{\mathrm{d}}{\mathrm{d} t} \widehat{\mathbf{a}}-\mathbf{G} \boldsymbol{\Phi}
$$

where $\mathbf{a}$ is the line-integrated magnetic vector potential and $\boldsymbol{\Phi}$ the electric scalar potential located on primary nodes. This is similar to the definition of the potentials in the continuous case, i.e., 1.15). The properties stated in this Lemma have been proven in [86, 8, 21].

The numbering scheme explained in Section 1.3.1 yields matrices with a simple banded structure. The sparsity pattern is such that an efficient implementation may not construct those matrices explicitly but apply the corresponding operations as such to vectors. However, the numbering scheme introduces superfluous objects allocated outside of the domain $\Omega$. For example in the case of the points located at the boundary where $i_{x}=n_{x}$, an edge in $x$ direction $L_{x}\left(n_{x}, i_{y}, i_{z}\right) \notin \Omega$. Those objects are called phantom objects. However, the homogeneous Dirichlet boundary conditions explained in Section 1.2.1, as well as the deletion of the phantom objects can be incorporated either by removing them with (truncated) projection matrices or by 
setting the corresponding degrees of freedom to zero. For a more detailed description of the process, see [8] and [86, Appendix A].

Assumption 4 (Boundary conditions) The degrees of freedom and all the operators are projected to an appropriate subspace considering the homogeneous Dirichlet boundary ('ebc') conditions and disregarding any phantom objects in $\widetilde{\mathbf{S}}, \widetilde{\mathbf{S}}^{\top}, \mathbf{C}$ and $\widetilde{\mathbf{C}}$. Therefore $\operatorname{ker} \widetilde{\mathbf{S}}^{\top}=0$.

This assumption imposes boundary conditions directly on the system matrices and thus is a necessary condition to ensure uniqueness of solution. It is important to note that the reduced matrices keep the properties described in Lemma 1, see for example [8, Section 3.2.4].

Please note that identical operators (without phantom objects) are obtained when applying the FE method with lowest-order Whitney basis functions using the same primal grid [21, 14].

\subsubsection{Material matrices}

The degrees of freedom have been introduced as integrals and thus the discretisation did not yet introduce any approximation error. This however happens when applying the matrices describing the material relations. In the FE case, the material matrices are given by the integrals

$$
\left[\mathbf{M}_{\xi}\right]_{n, m}=\int_{\Omega} \mathbf{w}_{n} \cdot \xi \mathbf{w}_{m} \mathrm{~d} \Omega
$$

where $\xi \in\{\boldsymbol{\sigma}, \boldsymbol{v}, \boldsymbol{\varepsilon}\}$ and $\mathbf{w}_{\star}$ are from an appropriate space, i.e., tangentially continuous Nédélec vectorial shape functions [72, 15] related to the $n$-th edge of the grid for discretising $\boldsymbol{\varepsilon}$ and $\boldsymbol{\sigma}$ and normally continuous Raviart-Thomas vectorial shape functions [78, 15] for discretizing $\boldsymbol{v}$.

In FIT, the matrix construction is derived from the Taylor expansion of the material laws. In the following, only the construction of the conductivity matrix is explained. For simplicity of notation, the conductivity $\sigma(\mathbf{r})$ is assumed to be isotropic and conforming to the primal grid, i.e. $\sigma^{(n)}=\sigma\left(\mathbf{r}_{n}\right)$ is constant on each primal volume $\left(\mathbf{r}_{n} \in V(n)\right)$. Consider a primal edge $L_{z}(i, j, k)$ and its associated dual facet $\widetilde{A}_{z}(i, j, k)$ (Figure 1.4. The tangential component $E_{z}$ of the electric field strength is continuous along $L_{z}(i, j, k)$ and is found by approximation from

$$
\widehat{\mathbf{e}}_{z}(i, j, k)=\int_{L_{z}(i, j, k)} \mathbf{E} \cdot \mathrm{d} \mathbf{s} \approx E_{z}\left|L_{z}(i, j, k)\right|,
$$

where $|\cdot|$ denotes the length, area or volume depending on the object. The current density integrated on the corresponding dual facet reads 


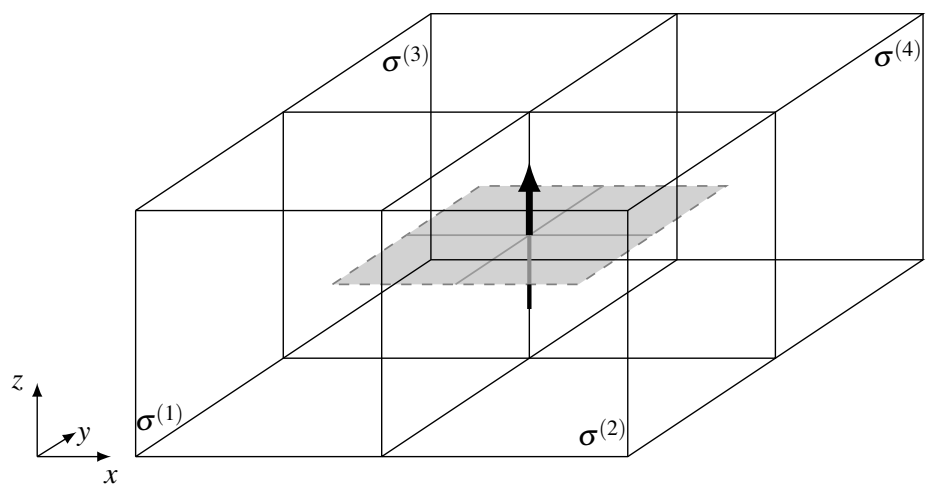

Fig. 1.4 Sketch of dual facet $\widetilde{A}_{z}(i, j, k)$ with its normal vector.

$$
\begin{aligned}
\widehat{\mathbf{j}}_{z}(i, j, k) & =\int_{\widetilde{A}_{z}(i, j, k)} \mathbf{J} \cdot \mathrm{d} \mathbf{A}=\int_{\widetilde{A}_{z}(i, j, k)} J_{z} \mathrm{~d} A=\sum_{q=1}^{4} \int_{\widetilde{A}_{z}^{(q)}(i, j, k)} \sigma^{(q)} E_{z} \mathrm{~d} A \\
& \approx \sum_{q=1}^{4} \sigma^{(q)} E_{z}\left|\widetilde{A}_{z}^{(q)}(i, j, k)\right|=\mathbf{M}_{\sigma, i, j, k} \widehat{\mathbf{e}}_{z}(i, j, k),
\end{aligned}
$$

where the conductances $\mathbf{M}_{\sigma, i, j, k}=\overline{\boldsymbol{\sigma}}(i, j, k) \frac{\left|\widetilde{A}_{z}(i, j, k)\right|}{\left|L_{z}(i, j, k)\right|}$ include the conductivities

$$
\bar{\sigma}(i, j, k)=\sum_{q=1}^{4} \sigma^{(q)} \frac{\left|\widetilde{A}_{z}^{(q)}(i, j, k)\right|}{\left|\widetilde{A}_{z}(i, j, k)\right|}
$$

averaged according to the conductivities $\sigma^{(q)}$ of the primal grid cells $V^{(q)}$ surrounding $L_{z}(i, j, k)$ and the surface fractions $\widetilde{A}_{z}^{(q)}(i, j, k)=V^{(q)} \cap \widetilde{A}_{z}(i, j, k)$. Analogously, material matrices for $\varepsilon$ and $v$ are obtained, which lead to the discretised material relations

$$
\widehat{\mathbf{d}}=\mathbf{M}_{\varepsilon} \widehat{\mathbf{e}}, \quad \widehat{\mathbf{j}}_{\mathrm{c}}=\mathbf{M}_{\sigma} \widehat{\mathbf{e}}, \quad \widehat{\mathbf{h}}=\mathbf{M}_{v} \widehat{\mathbf{b}}
$$

and

$$
\widehat{\mathbf{j}}=\widehat{\mathbf{j}}_{\mathrm{c}}+\widehat{\mathbf{j}}_{\mathrm{s}}
$$

with the source current density $\widehat{\mathbf{j}}_{s}$, which may be given by the discretisation $\mathbf{X}$ of the winding function 1.7 , such that $\widehat{\mathbf{j}}_{\mathrm{s}}=\sum_{k} \mathbf{X}_{k} i_{k}$ with currents $i_{k}$.

For the material matrices, one can show the following result [86, Appendix A]. 


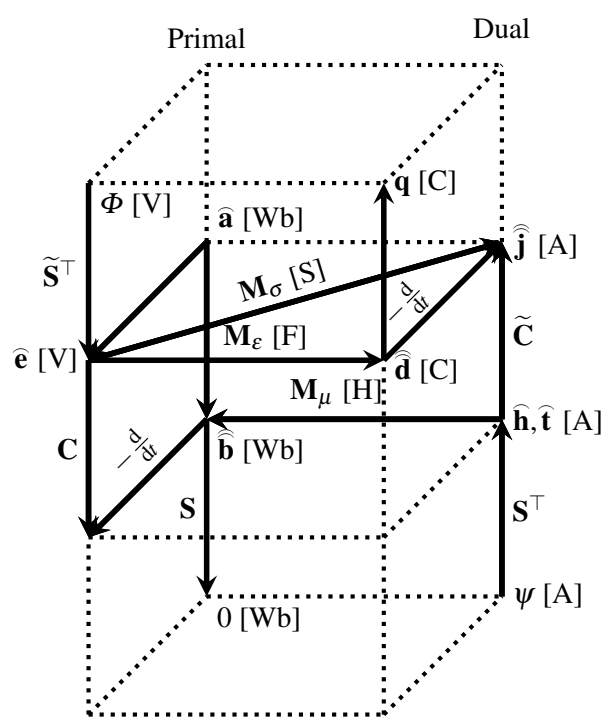

Fig. 1.5 Maxwell's house after spatial discretisation.

Lemma 2 (Material Matrices) The material matrices $\mathbf{M}_{\xi}$ are symmetric for all material properties $\xi=\{\sigma, v, \varepsilon\}$. If Assumption 2 holds, then the matrices $\mathbf{M}_{v}, \mathbf{M}_{\varepsilon}$ are positive definite whereas $\mathbf{M}_{\sigma}$ is only positive semidefinite.

Finally, the discretised version of Maxwell's equations with its corresponding material laws can be visualised by 'Maxwell's house' shown in Figure 1.5

Remark 3. Both Lemma 1 and 2, as well as Assumption 4 hold for Finite Element discretisations with basis functions fulfilling a discrete de Rham sequence.

Remark 4. In many applications the material parameters, e.g. the reluctivity, in 1.5 dependent nonlinearly on the fields. In these cases one may consider the linearised system but since the differential material properties inherit the relevant properties, e.g. [52], the characteristics of the DAE will also remain the same. In particular, there will be no change of nullspaces, see e.g. [7]

\subsection{Differential Algebraic Equations}

Starting from Maxwell's grid equations, various discrete time-domain formulations can be obtained. Depending on the choices made according to Definition 11, the resulting system is either static, first or second-order in time. In the dynamic cases, it can be written as a (linear) problem of the form

$$
\mathbf{M} \frac{\mathrm{d}}{\mathrm{d} t} \mathbf{x}(t)+\mathbf{K} \mathbf{x}(t)=\mathbf{r}(t), \quad \text { and } \quad \mathbf{x}\left(t_{0}\right)=\mathbf{x}_{0}
$$


where $\mathbf{M}, \mathbf{K} \in \mathbb{R}^{n \times n}$ are matrices, $\mathbf{x}:\left[t_{0}, T\right] \rightarrow \mathbb{R}^{n}$ contains the time-dependent degrees of freedom and $\mathbf{r}:\left[t_{0}, T\right] \rightarrow \mathbb{R}^{n}$ is an input.

Definition 2 (DAE). Equation (1.24) is called a system of differential-algebraic equations (DAE) if $\mathbf{M}$ is singular.

There are many options how to perform time-discretisation ('integration') of a DAE [1.24), see for example [47]. We suggest the simplest approach: implicit Euler's method, i.e.,

$$
(\mathbf{M} / \Delta t+\mathbf{K}) \mathbf{x}_{n+1}=\mathbf{r}\left(t_{n+1}\right)+\mathbf{M} / \Delta t \mathbf{x}_{n}
$$

where $\mathbf{x}_{n} \doteq \mathbf{x}\left(t_{n}\right)$ and $\Delta t=t_{n+1}-t_{n}$ is the time step. DAEs are commonly classified according to their index. Intuitively, it can be seen as a measure of the equations' sensitivity to perturbations of the input and the numerical difficulties when integrating. There are several competing index concepts. They essentially agree in the case of regular, linear problems, see [64] for detailed discussion. Therefore, we employ the simplest concept

Definition 3 (Differential Index, [22]). If solvable and the right-hand-side is smooth enough, then the DAE (1.24) has differential index- $\vartheta$ if $\vartheta$ is the minimal number of analytical differentiations with respect to the time $t$ that are necessary to obtain an ODE for $d \mathbf{x} / d t$ as a continuous function in $\mathbf{x}$ and $t$ by algebraic manipulations only.

For $\vartheta \geq 2$ the time-integration becomes difficult. Let us consider the classical educational index-2 problem to motivate analytically the sensitivity with respect to perturbations. The problem is described by

$$
\frac{\mathrm{d}}{\mathrm{d} t} x_{1}=x_{2} \quad \text { and } \quad x_{1}=\sin (t)+\delta(t)
$$

where $\delta(t)=10^{-k} \sin \left(10^{2 k} t\right)$ is a small perturbation with $k \gg 1$. The solution $x_{2}=$ $\cos (t)+10^{k} \cos \left(10^{2 k} t\right)$ is easily obtained by the product and chain rules. It shows that a very small perturbation in an index-2 system (at a high frequency) can have a serious impact (in the order of $10^{k}$ ) on the solution when compared to the original solution $x_{2}=\cos (t)$ of the unperturbed problem where $\delta=0$.

Remark 5. For the index analysis in the following sections we assume that the righthand sides are smooth enough.

Furthermore, DAEs are known for the fact that solutions have to fulfil certain constraints. One of the difficult parts in solving DAEs numerically is to determine a consistent set of initial conditions in order to start the integration [43, 69, 10].

Remark 6. [60] A vector $\mathbf{x}_{0} \in \mathbb{R}^{n}$ is called a consistent initial value if there is a solution of (1.24) through $\mathbf{x}_{0}$ at time $t_{0}$.

The problems discussed in the following will have at most (linear) index-2 components. For this case it has be shown that if we are not interested in a consistent 
initialisation at time $t_{0}$ but accept a solution satisfying the DAE only after the first step, then one may apply the implicit Euler method starting with an operating point and still obtain the same solution after $t>t_{0}$ that one would have obtained using a particular consistent value [10, 8].

The aim of this paper is to study the index of the systems obtained with different formulations and approximations according to Definition 1.

\subsection{Full-Wave Formulation}

On first sight it seems optimal to analyse high-frequency electromagnetic phenomena, e.g. the radiation of antennas, in frequency domain. The right-hand-sides can often be assumed to vary sinusoidally and for a given frequency, the equations are linear as the materials are rather frequency than field-dependent. However, the solution of problems in frequency domain requires the resolution of very large systems of equations and becomes inconvenient if one is interested in many frequencies (broadband solution). Therefore, often time-domain simulations are carried out with right-hand-sides that excite a large frequency spectrum.

\subsubsection{First-Order Formulation Time-Stepped by Leapfrog}

When solving Maxwell's grid equations for lossless $(\boldsymbol{\sigma} \equiv 0)$ wave propagation problems in time domain, a problem formulation based on the electric and magnetic field is commonly proposed. Assuming that the initial conditions fulfil the divergence relations of System (1.2), one starts with Faraday's and Ampère's laws

$$
\frac{\partial \mathbf{B}}{\partial t}+\nabla \times \mathbf{E}=0 \quad \text { and } \quad \frac{\partial \mathbf{D}}{\partial t}-\nabla \times \mathbf{H}=\mathbf{J}_{\mathrm{s}} .
$$

After inserting the material laws, the system becomes

$$
\boldsymbol{\mu} \frac{\partial \mathbf{H}}{\partial t}+\nabla \times \mathbf{E}=0 \quad \text { and } \quad \boldsymbol{\varepsilon} \frac{\partial \mathbf{E}}{\partial t}-\nabla \times \mathbf{H}=\mathbf{J}_{\mathrm{s}}
$$

with $\boldsymbol{v}=\boldsymbol{\mu}^{-1}$. Using Maxwell's grid equations (1.19), the semi-discrete initial value problem (IVP) has the form of equation 1.24) with unknown voltages $\mathbf{x}^{\top}:=$ $\left[\widehat{\mathbf{h}}^{\top}, \widehat{\mathbf{e}}^{\top}\right]$, right-hand-side $\mathbf{r}^{\top}:=\left[0, \widehat{\mathbf{j}}_{\mathrm{s}}^{\top}\right]$ and matrices

$$
\mathbf{M}:=\left[\begin{array}{cc}
\mathbf{M}_{v}^{-1} & 0 \\
0 & \mathbf{M}_{\varepsilon}
\end{array}\right] \text { and } \mathbf{K}:=\left[\begin{array}{cc}
0 & \mathbf{C} \\
-\widetilde{\mathbf{C}} & 0
\end{array}\right] .
$$

If Assumptions 2 and 4 holds, all superfluous degrees of freedom are removed and the material matrices $\mathbf{M}_{v}$ and $\mathbf{M}_{\varepsilon}$ have full rank. With FIT the matrices are further- 


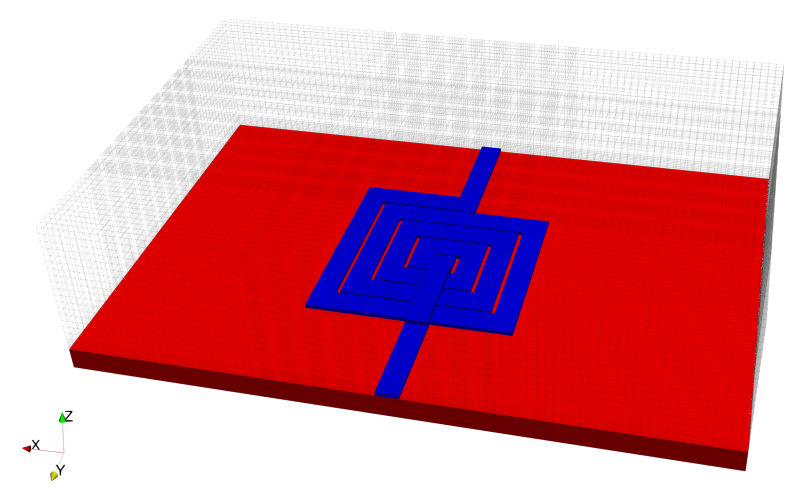

Fig. 1.6 Spiral inductor model with coplanar lines located on a substrate layer with an air bridge (Benchmark1)

more diagonal and thus easily inverted. A transformation by the matrices $\mathbf{M}_{v}^{-1 / 2}$ and $\mathbf{M}_{\mathcal{\varepsilon}}^{1 / 2}$ allows us to rewrite (1.24) as

$$
\frac{\mathrm{d}}{\mathrm{d} t} \overline{\mathbf{x}}(t)=\overline{\mathbf{K}} \overline{\mathbf{x}}(t)+\overline{\mathbf{r}}(t) \quad \overline{\mathbf{x}}\left(t_{0}\right)=\overline{\mathbf{x}}_{0}
$$

in the new unknowns $\overline{\mathbf{x}}^{\top}=\left[\left(\mathbf{M}_{v}^{-1 / 2} \widehat{\mathbf{h}}\right)^{\top},\left(\mathbf{M}_{\mathcal{\varepsilon}}^{1 / 2} \widehat{\mathbf{e}}\right)^{\top}\right]$ with the skew-symmetric stiffness matrix

$$
\overline{\mathbf{K}}=\left[\begin{array}{cc}
0 & -\mathbf{M}_{v}^{1 / 2} \mathbf{C} \mathbf{M}_{\varepsilon}^{-1 / 2} \\
\mathbf{M}_{\varepsilon}^{-1 / 2} \widetilde{\mathbf{C}} \mathbf{M}_{v}^{1 / 2} & 0
\end{array}\right]
$$

and right-hand-side $\overline{\mathbf{r}}^{\top}=\left[0,\left(\mathbf{M}_{\mathcal{\varepsilon}}^{-1 / 2} \widehat{\mathbf{j}}_{\mathrm{s}}\right)^{\top}\right]$. Let us conclude this by the following result

Theorem 1. Let Assumptions 1,2 and 4 hold. Then, the semidiscrete full-wave Maxwell equations expressed in the field strengths, i.e., (1.28) are an explicit system of ordinary differential equations.

The resulting IVP could be readily solved by the implicit Euler method 1.25 or any method that is tailored for second order differential equations. However, as explained above FIT allows to efficiently invert the mass matrix $\mathbf{M}$ and thus explicit methods become interesting. Typically the leapfrog scheme (or equivalently Störmer-Verlet) are used [91]. The restriction on the time step size related to the Courant-Friedrichs-Lewy-condition (CFL) is tolerable if the dynamics of the righthand-side are in a similar order of magnitude. Leapfrog is second-order accurate 
and symplectic, which is particularly interesting if there is no damping, i.e., no conductors present $(\boldsymbol{\sigma} \equiv 0)$. Furthermore it can be shown that space and time errors are well balanced when using the leapfrog scheme with the a time step size close to the CFL limit ("magic time step") [90, Chapters 2.4 and 4].

Let the initial conditions be

$$
\widehat{\mathbf{e}}^{(0)}=\widehat{\mathbf{e}}_{0} \quad \text { and } \quad \widehat{\mathbf{h}}^{\left(\frac{1}{2}\right)}=\widehat{\mathbf{h}}_{1 / 2}
$$

then the update equations for the leapfrog scheme read [97, 99, 90]

$$
\begin{aligned}
\widehat{\mathbf{e}}^{(m+1)} & =\widehat{\mathbf{e}}^{(m)}+\Delta t \mathbf{M}_{\mathcal{\varepsilon}}^{-1}\left(\widetilde{\mathbf{C}} \widehat{\mathbf{h}}^{\left(m+\frac{1}{2}\right)}-\widehat{\mathbf{j}}^{\left(m+\frac{1}{2}\right)}\right), \\
\widehat{\mathbf{h}}^{\left(m+\frac{3}{2}\right)} & =\widehat{\mathbf{h}}^{\left(m+\frac{1}{2}\right)}-\Delta t \mathbf{M}_{v} \widetilde{\mathbf{C}} \widehat{\mathbf{e}}^{(m+1)}
\end{aligned}
$$

for the electric and magnetic voltages $\widehat{\mathbf{e}}^{(m)}, \widehat{\mathbf{h}}^{\left(m+\frac{1}{2}\right)}$ at time instants $t_{m}$ and $t_{m+\frac{1}{2}}$ with step size $\Delta t$. For equidistant grids, the resulting scheme is (up to scaling and interpretation) equivalent to Yee's FDTD scheme [100].

Remark 7. In practice, one may choose to violate Assumption 4 Instead one imposes the boundary conditions by setting the corresponding entries in the material matrices $\mathbf{M}_{\varepsilon}^{-1}$ and $\mathbf{M}_{v}$ to zero. In this case the system (1.27) comes with additional (trivial) equations when compared to a system that is projected to the lower dimensional subspace containing the boundary conditions. However, this preserves a simpler structure of the equation system and the topological grid operators, e.g. the discrete curl matrix $\mathbf{C}$, keep their banded structure.

Benchmark 1 In [11] a spiral inductor model with coplanar lines located on a substrate layer with an air bridge was proposed as a benchmark example for highfrequency problems. The CST Microwave tutorial discusses the same model to advocate the usage of $3 D$ field simulation instead of circuit models [34]. A slightly simplified geometry is illustrated in Figure 1.6. The dimensions of the layer are $7 \cdot 10^{-4} \mathrm{~m} \times 4.75 \cdot 10^{-4} \mathrm{~m} \times 2.5 \cdot 10^{-5} \mathrm{~m}$ and Figure 1.7 illustrates the dimensions of the coil.

The bottom of the substrate layer is constrained by ebc and the other five boundaries are by mbc. On each side of the bridge the coil is connected by a straight line of perfect conductor with the ebc bottom plane. One side is excited by a discrete port which is given by a current source $i(t)=\sin (2 \pi f t) \mathrm{A}$ with $f=50 \cdot 10^{9} 1 / \mathrm{s}$. The coil $\left(\Omega_{\mathrm{pec}}\right)$ is assumed to be a perfect conductor, i.e., modelled by homogeneous electric boundary conditions, the substrate $\left(\Omega_{\mathrm{sub}}\right)$ is given a relative permittivity of $\varepsilon_{r}=12$, in the air region $\Omega_{\mathrm{air}} \varepsilon_{r}=1$ and vacuum permeability $\mu=4 \pi \cdot 1 \cdot 10^{-7} \mathrm{H} / \mathrm{m}$ is assumed everywhere else.

The structure is discretised using FIT with 406493 mesh cells and 1, 283,040 degrees of freedom. Leapfrog is used with a time step of $\Delta t=3.4331 \cdot 10^{-15} \mathrm{~s}$ based 


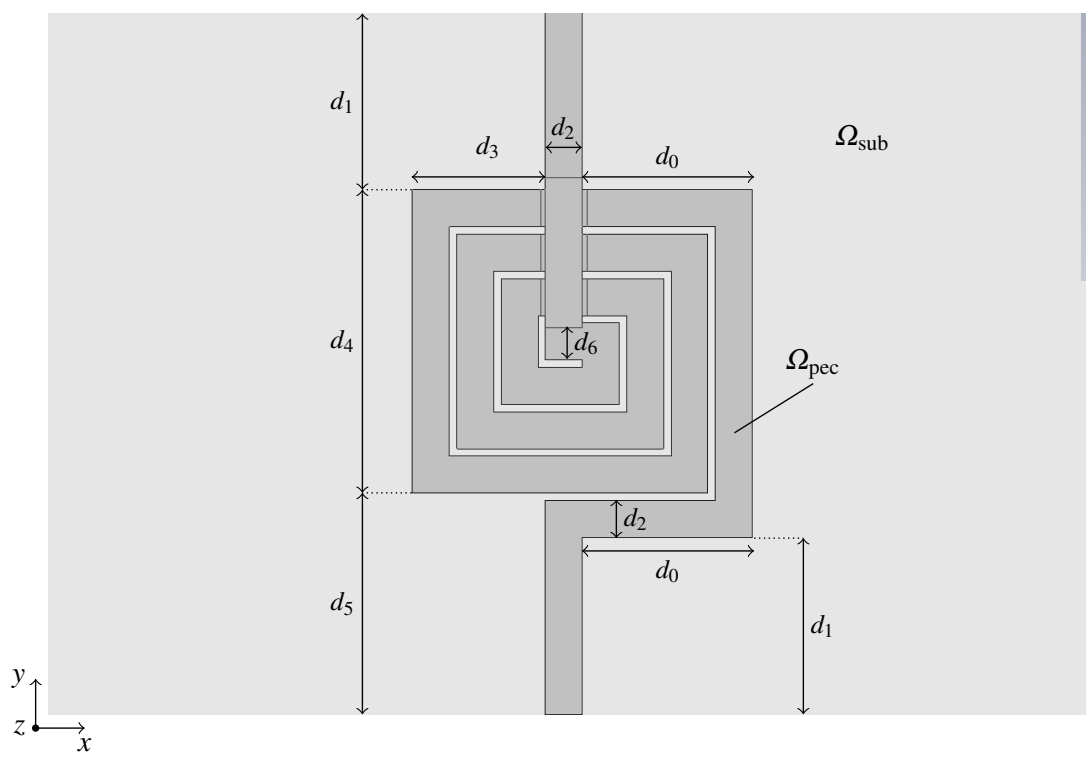

(a) $x-y$ cross section of spiral inductor.

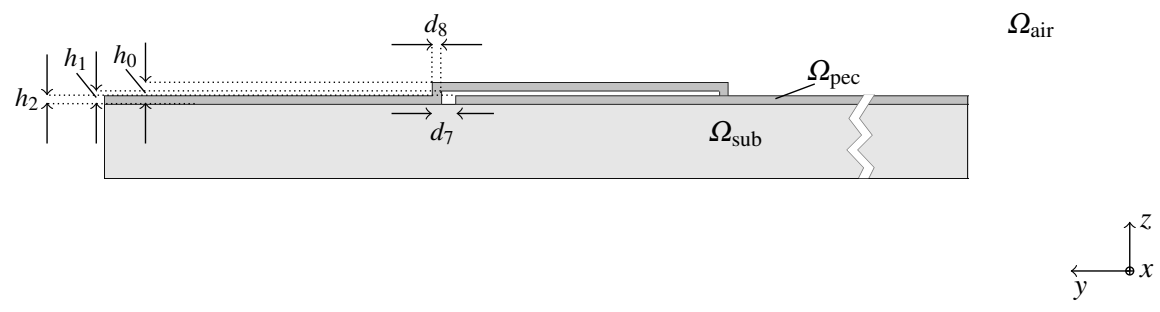

(b) $y-z$ cross section of spiral inductor.

Fig. 1.7 Model of a spiral inductor of Benchmark 1 The distances are $d_{0}=1.15 \cdot 10^{-4} \mathrm{~m}, d_{1}=1.2$. $10^{-4} \mathrm{~m}, d_{2}=2.5 \cdot 10^{-5} \mathrm{~m}, d_{3}=9 \cdot 10^{-5} \mathrm{~m}, d_{4}=2.05 \cdot 10^{-4} \mathrm{~m}, d_{5}=1.5 \cdot 10^{-4} \mathrm{~m}, d_{6}=2.2 \cdot 10^{-5} \mathrm{~m}$, $d_{7}=9 \cdot 10^{-6} \mathrm{~m}, d_{8}=3 \cdot 10^{-6} \mathrm{~m}, h_{0}=8 \cdot 10^{-6} \mathrm{~m}, h_{1}=5 \cdot 10^{-6} \mathrm{~m}$ and $h_{2}=3 \cdot 10^{-6} \mathrm{~m}$.

on the CFL condition and zero initial condition, see Fig. 1.8 The performance of leapfrog and exponential integrators for this model was recently discussed in [65].

\subsubsection{A- $\Phi$ formulations}

If there are small geometric features, slowly varying excitations, conducting or semiconducting materials [27], the leapfrog scheme becomes inefficient. An alternative formulation is obtained if one rewrites Maxwell's equations as a second-order 


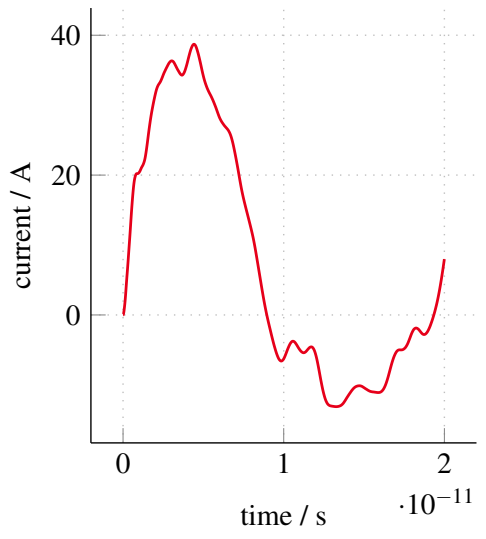

(a) Current through the spiral inductor

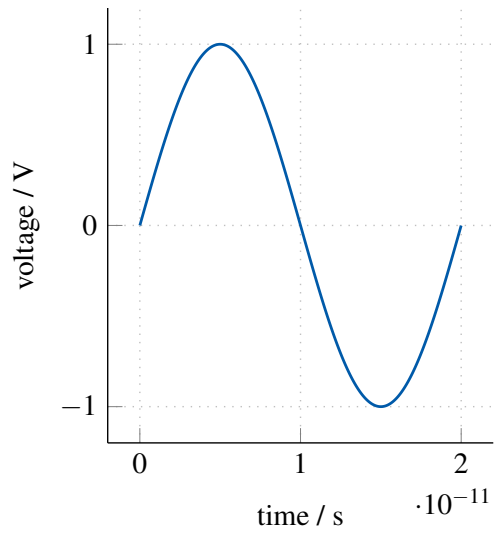

(b) voltage drop at the ports

Fig. 1.8 Time domain simulation results for the Benchmark 1

partial differential equation by combining Faraday's law, Ampère's law and the material equations complemented by Gauss's law, i.e.,

$$
\begin{aligned}
\boldsymbol{\varepsilon} \frac{\partial^{2}}{\partial t^{2}} \mathbf{E}+\boldsymbol{\sigma} \frac{\partial}{\partial t} \mathbf{E}+\nabla \times v \nabla \times \mathbf{E} & =\frac{\partial}{\partial t} \mathbf{J}_{\mathrm{s}} . \\
-\nabla \cdot \boldsymbol{\varepsilon} \mathbf{E} & =\rho
\end{aligned}
$$

The inconvenience of a time-differentiated source current density can be mitigated by exploiting the potentials as defined in 1.15

$$
\begin{aligned}
\boldsymbol{\varepsilon} \frac{\partial^{2}}{\partial t^{2}} \mathbf{A}+\frac{\partial}{\partial t} \boldsymbol{\varepsilon} \nabla \phi+\boldsymbol{\sigma} \frac{\partial}{\partial t} \mathbf{A}+\boldsymbol{\sigma} \nabla \phi+\nabla \times v \nabla \times \mathbf{A}=\mathbf{J}_{\mathrm{s}} \\
-\nabla \cdot \boldsymbol{\varepsilon} \frac{\partial}{\partial t} \mathbf{A}-\nabla \cdot \boldsymbol{\varepsilon} \nabla \phi=\rho .
\end{aligned}
$$

There is an ambiguity of the electromagnetic potentials since $\mathbf{A}$ is only fixed up to a gradient field [54]. To this end, several gauging techniques have been introduced. For example grad-div formulations that are based on the Coulomb gauge, have been introduced for low frequencies [19, 31, 29] and high-frequency applications [46].

Let us define a regularisation of the electrodynamic potentials by the following gauge condition

$$
\xi_{1} \nabla \cdot \mathbf{A}+\xi_{2} \phi+\xi_{3} \frac{\partial}{\partial t} \phi=0
$$

which yields for $\xi_{1}=1, \xi_{2}=\xi_{3}=0$ the Coulomb gauge and for $\xi_{1}=v, \xi_{2}=0$ and $\xi_{3}=\varepsilon$ the Lorenz gauge if the considered materials are conducting, uniform, isotropic and linear. In the case $\xi_{1}=v, \xi_{2}=\sigma$ and $\xi_{3}=\varepsilon$ the curl-curl equation 
(1.32) can be written as a pair of damped wave equations

$$
\begin{aligned}
& {\left[\Delta-\mu \sigma \frac{\partial}{\partial t}-\mu \varepsilon \frac{\partial^{2}}{\partial t^{2}}\right] \mathbf{A}=-\mu \mathbf{J}_{\mathrm{s}}} \\
& {\left[\Delta-\mu \sigma \frac{\partial}{\partial t}-\mu \varepsilon \frac{\partial^{2}}{\partial t^{2}}\right] \phi=-\frac{\rho}{\varepsilon}}
\end{aligned}
$$

where $\Delta$ denotes the (scalar and vector) Laplace operators. In the undamped case $(\sigma=0)$, this system reduces to the well-known d'Alembert equations, [54]. The right-hand-sides are still coupled via the continuity equation 1.3

$$
\nabla \cdot \mathbf{J}_{\mathrm{s}}+\frac{\sigma}{\varepsilon} \rho+\frac{\partial}{\partial t} \rho=0
$$

where we have again exploited isotropy and homogeneity of $\sigma$ and $\varepsilon$ to obtain $-\nabla \cdot \sigma \mathbf{E}=\frac{\sigma}{\varepsilon} \rho$. When solving the system (1.35)-(1.37) we have to ensure that the (generalised) Lorenz gauge (1.34) is still fulfilled, which requires compatible boundary conditions for $\mathbf{A}$ and $\phi[9]$.

Now, let us derive a similar semidiscrete formulation based on the spatial discretisation introduced above. We start with the $A-\phi$ formulation (1.32) using the discretised laws of Ampère (1.19b) and Gauss $1.19 \mathrm{~d}$ ):

$$
\begin{aligned}
\tilde{\mathbf{C}} \mathbf{M}_{v} \mathbf{C} \widehat{\mathbf{a}}+\mathbf{M}_{\sigma}\left[\frac{\mathrm{d}}{\mathrm{d} t} \widehat{\mathbf{a}}+\mathbf{G} \boldsymbol{\Phi}\right]+\mathbf{M}_{\varepsilon}\left[\frac{\mathrm{d}^{2}}{\mathrm{~d} t^{2}} \widehat{\mathbf{a}}+\mathbf{G} \frac{\mathrm{d}}{\mathrm{d} t} \boldsymbol{\Phi}\right] & =\widehat{\mathbf{j}}_{\mathrm{s}} \\
-\tilde{\mathbf{S}} \mathbf{M}_{\varepsilon} \frac{\mathrm{d}}{\mathrm{d} t} \widehat{\mathbf{a}}+\mathbf{L}_{\varepsilon} \boldsymbol{\Phi} & =\mathbf{q}
\end{aligned}
$$

which contains the discrete Laplace operators

$$
\mathbf{L}_{\varepsilon}:=-\tilde{\mathbf{S}} \mathbf{M}_{\varepsilon} \mathbf{G} \quad \text { and } \quad \mathbf{L}_{\sigma}:=-\tilde{\mathbf{S}} \mathbf{M}_{\sigma} \mathbf{G},
$$

for permittivity and conductivity, respectively.

Lemma 3 (Discrete Laplacians) Let Assumptions 2 and 4 hold true, the discrete Laplace operator $\mathbf{L}_{\varepsilon}$ in 1.40 is symmetric positive definite and $\mathbf{L}_{\sigma}$ is symmetric positive semidefinite.

Proof. As we assume Dirichlet boundary conditions (ebc) in Assumption 4 and $\mathbf{G}=-\tilde{\mathbf{S}}^{\top}$ due to 1.21 , the proof is straight forward.

Equations 1.38 and 1.39 are coupled by the potentials and right-hand-sides via the continuity equation

$$
\tilde{\mathbf{S}} \widehat{\mathbf{j}}_{\mathrm{s}}+\mathbf{L}_{\sigma} \mathbf{L}_{\varepsilon}^{-1} \mathbf{q}+\frac{\mathrm{d}}{\mathrm{d} t} \mathbf{q}=\left[\tilde{\mathbf{S}} \mathbf{M}_{\sigma}-\mathbf{L}_{\sigma} \mathbf{L}_{\varepsilon}^{-1} \tilde{\mathbf{S}} \mathbf{M}_{\varepsilon}\right] \frac{\mathrm{d}}{\mathrm{d} t} \widehat{\mathbf{a}},
$$


that is obtained by a left multiplication of Ampère's law by $\tilde{\mathbf{S}}$ and inserting Gauss' law etc. The steps are the same as in the continuous case, e.g., applying the divergence operator. Nonetheless, the discrete continuity equation (1.41) is more general than its continuous counterpart 1.37) as it covers anisotropic and non-homogeneous material distributions.

The ambiguity of the potentials is not yet fixed. The generalised discrete Lorenz gauge (1.34) for a conductive domain in FIT notation is given by

$$
\mathbf{M}_{\varepsilon} \mathbf{G M}_{\mathrm{N}} \tilde{\mathbf{S}} \mathbf{M}_{\varepsilon} \widehat{\mathbf{a}}+\mathbf{M}_{\sigma} \mathbf{G} \boldsymbol{\Phi}+\mathbf{M}_{\varepsilon} \mathbf{G} \frac{\mathrm{d}}{\mathrm{d} t} \boldsymbol{\Phi}=0
$$

with a scaling matrix $\mathbf{M}_{\mathrm{N}}$ which is mainly introduced to guarantee correct units. A consistent but rather inconvenient choice is

$$
\mathbf{M}_{\mathrm{N}}:=\mathbf{M}_{\varepsilon}^{-1 / 2} \mathbf{M}_{v}^{1 / 2} \mathbf{L}_{\varepsilon}^{-1} \mathbf{M}_{v}^{1 / 2} \mathbf{M}_{\varepsilon}^{-1 / 2}
$$

This regularisation is similar to the Lagrange-multiplier formulation for the eddycurrent problem, [28]. Left-multiplication of $\left[1.42\right.$ by $\mathbf{M}_{\mathrm{N}}^{-1} \mathbf{L}_{\varepsilon}^{-1} \tilde{\mathbf{S}}$ yields

$$
\tilde{\mathbf{S}} \mathbf{M}_{\varepsilon} \widehat{\mathbf{a}}+\mathbf{M}_{\mathrm{N}}^{-1} \mathbf{L}_{\varepsilon}^{-1} \mathbf{L}_{\sigma} \boldsymbol{\Phi}+\mathbf{M}_{\mathrm{N}}^{-1} \frac{\mathrm{d}}{\mathrm{d} t} \boldsymbol{\Phi}=0
$$

which simplifies to Coulomb's gauge

$$
\tilde{\mathbf{S}} \mathbf{M}_{\varepsilon} \widehat{\mathbf{a}}=0
$$

with respect to the permittivities if we set $\boldsymbol{\Phi}=0$.

To obtain a discrete version of the damped wave equation (1.35)-(1.36), we utilise (1.43). Now, using (1.42) and (1.43) the system (1.38)-(1.39) becomes two discrete damped wave equations

$$
\begin{array}{r}
\mathbf{L}_{v} \widehat{\mathbf{a}}+\mathbf{M}_{\sigma} \frac{\mathrm{d}}{\mathrm{d} t} \widehat{\mathbf{a}}+\mathbf{M}_{\varepsilon} \frac{\mathrm{d}^{2}}{\mathrm{~d} t^{2}} \widehat{\mathbf{a}}=\widehat{\mathbf{j}}_{\mathrm{s}} \\
\mathbf{L}_{\varepsilon} \boldsymbol{\Phi}+\mathbf{M}_{\mathrm{N}}^{-1} \mathbf{L}_{\varepsilon}^{-1} \mathbf{L}_{\sigma} \frac{\mathrm{d}}{\mathrm{d} t} \boldsymbol{\Phi}+\mathbf{M}_{\mathrm{N}}^{-1} \frac{\mathrm{d}^{2}}{\mathrm{~d} t^{2}} \boldsymbol{\Phi}=\mathbf{q}
\end{array}
$$

with $\mathbf{L}_{v}:=\widetilde{\mathbf{C}} \mathbf{M}_{v} \mathbf{C}-\mathbf{M}_{\varepsilon} \mathbf{G} \mathbf{M}_{\mathrm{N}} \widetilde{\mathbf{S}} \mathbf{M}_{\varepsilon}$ and given right-hand-sides $\widehat{\mathbf{j}}_{\mathrm{S}}$ and $\mathbf{q}$ that fulfil the continuity equation (1.41). The resulting problem 1.45)-(1.46) is a system of second-order ordinary differential equations:

Theorem 2. Let Assumptions 1,2 and 4 hold. Then, the A- $\Phi$-formulation with Lorenz gauge (1.43) and known charges $\mathbf{q}$ leads to an ordinary differential equation $(O D E)$ system which is given in 1.45-1.46. 


\subsubsection{Full Maxwell with Lorenz Gauge}

Let us now investigate the case where the charges $\mathbf{q}$ are not known. We start from Lorenz' gauge (1.42). Left-multiplication of the equation by $-\tilde{\mathbf{S}}$ yields

$$
\mathbf{L}_{\varepsilon} \mathbf{M}_{\mathrm{N}} \tilde{\mathbf{S}} \mathbf{M}_{\varepsilon} \widehat{\mathbf{a}}+\mathbf{L}_{\sigma} \boldsymbol{\Phi}+\mathbf{L}_{\varepsilon} \frac{\mathrm{d}}{\mathrm{d} t} \boldsymbol{\Phi}=0 .
$$

Following the notation of Schoenmaker, e.g. [83], we denote the derivative of the magnetic vector potential by $\pi:=\mathrm{d} \mathbf{a} / \mathrm{d} t$. Then, the equations (1.38)-11.39) can be rearranged as the following system of DAEs

$$
\begin{array}{r}
\mathbf{L}_{\varepsilon} \mathbf{M}_{\mathrm{N}} \tilde{\mathbf{S}} \mathbf{M}_{\varepsilon} \widehat{\mathbf{a}}+\mathbf{L}_{\sigma} \boldsymbol{\Phi}+\mathbf{L}_{\varepsilon} \frac{\mathrm{d}}{\mathrm{d} t} \boldsymbol{\Phi}=0 \\
\tilde{\mathbf{C}} \mathbf{M}_{v} \mathbf{C} \widehat{\mathbf{a}}+\mathbf{M}_{\sigma}[\widehat{\pi}+\mathbf{G} \boldsymbol{\Phi}]+\mathbf{M}_{\varepsilon}\left[\frac{\mathrm{d}}{\mathrm{d} t} \widehat{\pi}+\mathbf{G} \frac{\mathrm{d}}{\mathrm{d} t} \boldsymbol{\Phi}\right]=\widehat{\mathbf{j}}_{\mathrm{s}} \\
\tilde{\mathbf{S}} \mathbf{M}_{\varepsilon} \widehat{\pi}-\mathbf{L}_{\varepsilon} \boldsymbol{\Phi}+\mathbf{q}=0 \\
\frac{\mathrm{d}}{\mathrm{d} t} \widehat{\mathbf{a}}-\widehat{\pi}=0
\end{array}
$$

with $\mathbf{x}^{\top}=\left(\mathbf{q}^{\top}, \boldsymbol{\Phi}^{\top}, \widehat{\mathbf{a}}^{\top}, \hat{\pi}^{\top}\right)$ such that we can write (1.47)-(1.50) in the form of (1.24) with the definitions

$$
\mathbf{M}=\left[\begin{array}{cccc}
0 & \mathbf{L}_{\varepsilon} & 0 & 0 \\
0 & \mathbf{M}_{\varepsilon} \mathbf{G} & 0 & \mathbf{M}_{\varepsilon} \\
0 & 0 & 0 & 0 \\
0 & 0 & \mathbf{I} & 0
\end{array}\right], \quad \mathbf{K}=\left[\begin{array}{cccc}
0 & \mathbf{L}_{\sigma} & \mathbf{L}_{\varepsilon} \mathbf{M}_{\mathrm{N}} \tilde{\mathbf{S}} \mathbf{M}_{\varepsilon} & 0 \\
0 & \mathbf{M}_{\sigma} \mathbf{G} & \tilde{\mathbf{C}} \mathbf{M}_{v} \mathbf{C} & \mathbf{M}_{\sigma} \\
\mathbf{I} & -\mathbf{L}_{\varepsilon} & 0 & \tilde{\mathbf{S}} \mathbf{M}_{\varepsilon} \\
0 & 0 & 0 & -\mathbf{I}
\end{array}\right] \quad \text { and } \quad \mathbf{r}=\left[\begin{array}{c}
0 \\
\widehat{\mathbf{j}}_{\mathrm{s}} \\
0 \\
0
\end{array}\right]
$$

Now, any standard time integrator, e.g. the implicit Euler method 1.25, can be applied.

Next we determine the differential index of the system (1.47)-(1.50). Equation (1.47) is an ODE for $\boldsymbol{\Phi}$

$$
\frac{\mathrm{d}}{\mathrm{d} t} \boldsymbol{\Phi}=-\mathbf{M}_{\mathrm{N}} \tilde{\mathbf{S}} \mathbf{M}_{\varepsilon} \widehat{\mathbf{a}}-\mathbf{L}_{\varepsilon}^{-1} \mathbf{L}_{\sigma} \boldsymbol{\Phi} .
$$

Then, we deduce from (1.48) and (1.51) an ODE for $\pi$ :

$$
\frac{\mathrm{d}}{\mathrm{d} t} \widehat{\pi}=-\mathbf{M}_{\varepsilon}^{-1}\left[\mathbf{L}_{v} \widehat{\mathbf{a}}+\mathbf{M}_{\sigma}[\widehat{\pi}+\mathbf{G} \boldsymbol{\Phi}]-\mathbf{M}_{\varepsilon} \mathbf{G} \mathbf{L}_{\varepsilon}^{-1} \mathbf{L}_{\sigma} \boldsymbol{\Phi}-\widehat{\mathbf{j}}_{\mathrm{s}}\right]
$$

Finally, only one differentiation with respect to time of 1.49 is needed to obtain an ordinary differential equation for $\mathbf{q}$ :

$$
\frac{\mathrm{d}}{\mathrm{d} t} \mathbf{q}=\tilde{\mathbf{S}} \mathbf{M}_{\sigma} \widehat{\pi}-\mathbf{L}_{\sigma} \boldsymbol{\Phi}-\tilde{\mathbf{S}} \widehat{\mathbf{j}}_{\mathrm{s}}
$$


Hence we conclude the following result [9]

Theorem 3. Let Assumptions 1, 2 and 4 hold. The system (1.47)-(1.50) has differential index-1 and the initial vector $\mathbf{x}_{0}^{\top}=\left(\mathbf{q}_{0}^{\top}, \boldsymbol{\Phi}_{0}^{\top}, \widehat{\mathbf{a}}_{0}^{\top}, \widehat{\pi}_{0}^{\top}\right)$ is a consistent initial value if $\mathbf{q}_{0}=\mathbf{L}_{\varepsilon} \boldsymbol{\Phi}_{0}-\tilde{\mathbf{S}} \mathbf{M}_{\varepsilon} \widehat{\pi}_{0}$ is fulfilled.

\subsubsection{Full Maxwell with Coulomb Gauge}

Instead of augmenting the equations by a Lorenz gauge, one can choose the Coulomb gauge (1.44). Starting by left-multiplying Coulomb's gauge by $\mathbf{M}_{\varepsilon} \mathbf{G} \mathbf{M}_{\mathrm{N}}$, we obtain

$$
\mathbf{M}_{\varepsilon} \mathbf{G M}_{\mathrm{N}} \tilde{\mathbf{S}} \mathbf{M}_{\varepsilon} \widehat{\mathbf{a}}=0 .
$$

Using (1.44) and (1.52), the system (1.38)-(1.39) becomes a semi-discrete damped wave equation accompanied by a Laplace equation, i.e.,

$$
\begin{aligned}
\mathbf{L}_{v} \widehat{\mathbf{a}}+\mathbf{M}_{\sigma}\left[\frac{\mathrm{d}}{\mathrm{d} t} \widehat{\mathbf{a}}+\mathbf{G} \boldsymbol{\Phi}\right]+\mathbf{M}_{\varepsilon}\left[\frac{\mathrm{d}^{2}}{\mathrm{~d} t^{2}} \widehat{\mathbf{a}}+\mathbf{G} \frac{\mathrm{d}}{\mathrm{d} t} \boldsymbol{\Phi}\right] & =\widehat{\mathbf{j}}_{\mathrm{s}} \\
\mathbf{L}_{\varepsilon} \boldsymbol{\Phi} & =\mathbf{q}
\end{aligned}
$$

with right-hand-sides that fulfil the continuity equation (1.41) and thus for given $\widehat{\mathbf{j}}_{\mathrm{s}}$ the resulting semi-discrete problem is again a system of DAEs. The Coulombgauged system reads

$$
\begin{aligned}
\tilde{\mathbf{S}} \mathbf{M}_{\varepsilon} \widehat{\mathbf{a}} & =0 \\
\tilde{\mathbf{C}} \mathbf{M}_{v} \mathbf{C} \widehat{\mathbf{a}}+\mathbf{M}_{\sigma}[\tilde{\pi}+\mathbf{G} \boldsymbol{\Phi}]+\mathbf{M}_{\varepsilon}\left[\frac{\mathrm{d}}{\mathrm{d} t} \widehat{\pi}+\mathbf{G} \frac{\mathrm{d}}{\mathrm{d} t} \boldsymbol{\Phi}\right] & =\widehat{\mathbf{j}}_{\mathrm{s}} \\
\tilde{\mathbf{S}} \mathbf{M}_{\varepsilon} \widehat{\pi}-\mathbf{L}_{\varepsilon} \boldsymbol{\Phi}+\mathbf{q} & =0 \\
\frac{\mathrm{d}}{\mathrm{d} t} \widehat{\mathbf{a}}-\widehat{\pi} & =0
\end{aligned}
$$

with $\mathbf{x}^{\top}=\left(\mathbf{q}^{\top}, \boldsymbol{\Phi}^{\top}, \widehat{\mathbf{a}}^{\top}, \hat{\pi}^{\top}\right)$. Similarly as before we can identify a first order DAE system of form (1.24) and apply for example the implicit Euler method.

Next, we determine the differential index of the system (1.53)- $(1.56)$. Differentiating (1.53) twice with respect to time and inserting (1.56) leads to

$$
\tilde{\mathbf{S}} \mathbf{M}_{\varepsilon} \frac{\mathrm{d}}{\mathrm{d} t} \widehat{\pi}=0 .
$$

This indicates already that the differential index is at least $\vartheta \geq 2$. Left-multiplying 1.54 by $\tilde{\mathbf{S}}$ and applying 1.57 yields:

$$
\frac{\mathrm{d}}{\mathrm{d} t} \boldsymbol{\Phi}=-\mathbf{L}_{\varepsilon}^{-1}\left[\mathbf{L}_{\sigma} \boldsymbol{\Phi}-\tilde{\mathbf{S}} \mathbf{M}_{\sigma} \hat{\pi}+\tilde{\mathbf{S}} \widehat{\mathbf{j}}_{\mathrm{s}}\right]
$$




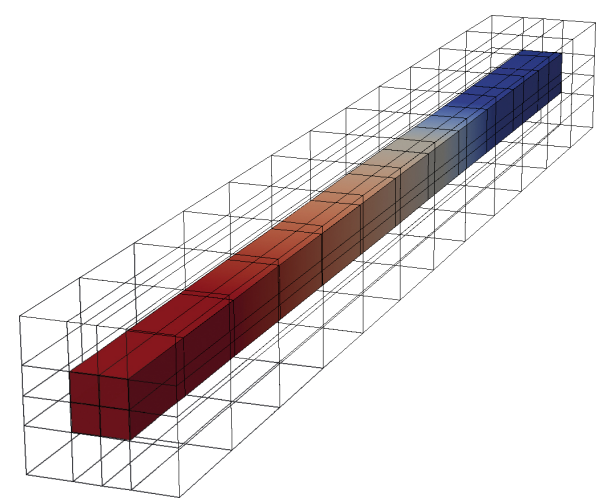

Fig. 1.9 Copper bar in air, excited by sinusoidal source (Benchmark 2

Furthermore, from (1.54), we obtain:

$$
\begin{aligned}
\frac{\mathrm{d}}{\mathrm{d} t} \tilde{\pi}= & -\mathbf{M}_{\varepsilon}^{-1}\left[\left(\mathbf{M}_{\sigma} \mathbf{G}-\mathbf{M}_{\varepsilon} \mathbf{G} \mathbf{L}_{\varepsilon}^{-1} \mathbf{L}_{\sigma}\right) \boldsymbol{\Phi}+\tilde{\mathbf{C}} \mathbf{M}_{\nu} \mathbf{C} \widehat{\mathbf{a}}\right. \\
& \left.+\left(\mathbf{M}_{\sigma}+\mathbf{M}_{\varepsilon} \mathbf{G} \mathbf{L}_{\varepsilon}^{-1} \tilde{\mathbf{S}} \mathbf{M}_{\sigma}\right) \tilde{\pi}-\left(\mathbf{I}+\mathbf{M}_{\varepsilon} \mathbf{G} \mathbf{L}_{\varepsilon}^{-1} \tilde{\mathbf{S}}\right) \widehat{\mathbf{j}}_{\mathrm{s}}\right]
\end{aligned}
$$

Finally, one differentiation with respect to time of (1.55) results in

$$
\frac{\mathrm{d}}{\mathrm{d} t} \mathbf{q}=\tilde{\mathbf{S}} \mathbf{M}_{\sigma} \tilde{\pi}-\mathbf{L}_{\sigma} \boldsymbol{\Phi}-\tilde{\mathbf{S}} \widehat{\mathbf{j}}_{\mathrm{s}}
$$

and thus the overall problem has a differential index-2 [9].

Theorem 4. Let Assumptions 1,2 and 4 hold. The system (1.53)-(1.56) has differential index-2 and the initial vector $\mathbf{x}_{0}^{\top}=\left(\mathbf{q}_{0}^{\top}, \boldsymbol{\Phi}_{0}^{\top}, \mathbf{a}_{0}^{\top}, \widehat{\pi}_{0}^{\top}\right)$ is a consistent initial value if $\tilde{\mathbf{S}} \mathbf{M}_{\varepsilon} \widehat{\mathbf{a}}_{0}=0, \tilde{\mathbf{S}} \mathbf{M}_{\varepsilon} \widehat{\pi}_{0}=0$ and $\mathbf{q}_{0}=\mathbf{L}_{\varepsilon} \boldsymbol{\Phi}_{0}-\tilde{\mathbf{S}} \mathbf{M}_{\varepsilon} \widehat{\pi}_{0}$ are fulfilled.

Lorenz' and Coulomb's gauge lead to systems that describe the same phenomena and have eventually, i.e. in the mesh size limit, the same electromagnetic fields (strengths or fluxes) as solutions. On the other hand, the structural properties are different, i.e., the Lorenz gauge yields an index-1 problem whereas the Coulomb gauge gives index-2. Hence, the latter formulation will be much more affected by perturbations and the computation of consistent initial values is more cumbersome. This has been observed in simulations [9, 8].

Benchmark 2 The benchmark example Fig. 1.9 was proposed in [9] to numerically analyse the DAE index of the two gauged A- $\phi$ formulations. The model is a copper bar with a cross-sectional area of $0.25 \mathrm{~mm}^{2}$ surrounded by air and discretised by FIT. A detailed characterisation of the dimensions can be seen in Figure 1.10

On the copper bar $\Omega_{\mathrm{c}}$, a conductivity of $\sigma_{\mathrm{c}}=5.7 \cdot 10^{7} \mathrm{~S} / \mathrm{m}$ is set and on the air region $\sigma_{\mathrm{air}}=0 \mathrm{~S} / \mathrm{m}$. Vacuum permeability $\mu=4 \pi \cdot 10^{-7} \mathrm{H} / \mathrm{m}$ and relative permittivity $\varepsilon_{r}=1$ is assumed in the entire domain. 


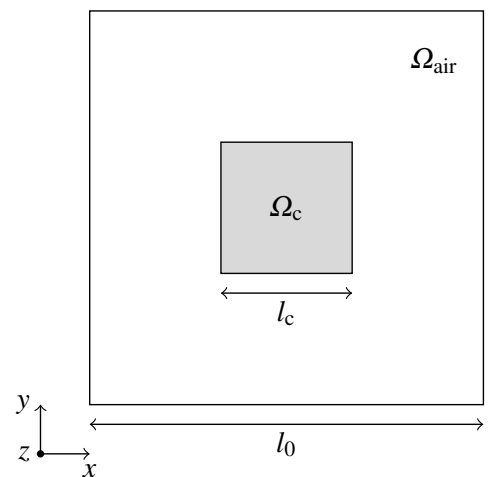

(a) Squared cross-section of the copper bar at the $\mathrm{z}=0$ plane.

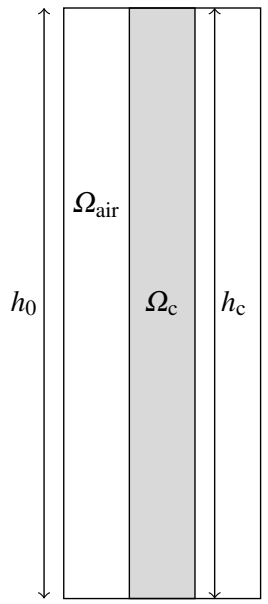

(b) Cross-section of the copper bar at the $\mathrm{x}=0$ plane.

Fig. 1.10 Copper bar with square cross-section surrounded by air. The distances are $l_{\mathrm{c}}=(1+\sqrt{1.5}) \cdot 10^{-3} \mathrm{~m}, l_{0}=(3+\sqrt{1.5}) \cdot 10^{-3} \mathrm{~m}$, and $h_{0}=h_{\mathrm{c}}=3 \mathrm{~m}$.

One contact is excited by a sinusoidal voltage $v=\sin (2 \pi t) \mathrm{V}$, the other contact is grounded (ebc) and the remaining boundary is set to $m b c$.

The structure is discretised using FIT with 325 mesh cells and 845 degrees of freedom. The implicit Euler method is applied with a time step of $\Delta t=1 \cdot 10^{-4} \mathrm{~s}$ and zero initial condition, see Fig. 1.11

\subsection{Quasistatic Maxwell's Equations}

In the case of slowly time-varying fields, certain time-derivatives of Maxwell's equations can be disregarded with respect to other phenomena, see Definition 11 This is convenient to simplify the numerical treatment. However, the resulting (quasi-) static approximations have different structural properties and their differential algebraic index is studied next.

\subsubsection{Electroquasistatic Maxwell's Equations}

We start with the index study of the electroquasistatic approximation, that is given in Definition $1 \mathrm{~b}$ ). Maxwell's equations can be rewritten as 


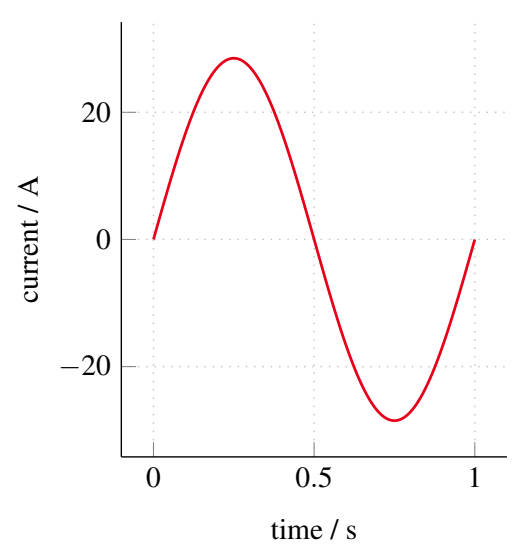

(a) Current through the bar

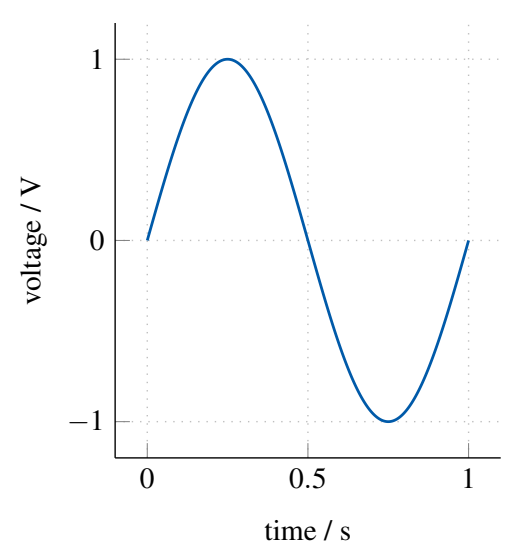

(b) voltage drop at the ports

Fig. 1.11 Time domain simulation results for the Benchmark 2 with Lorenz gauge $\Delta t=1 \cdot 10^{-4} \mathrm{~s}$.

$$
\nabla \times \mathbf{E}=0, \quad \nabla \times \mathbf{H}=\frac{\partial \mathbf{D}}{\partial t}+\mathbf{J}, \quad \nabla \cdot \mathbf{D}=\rho, \quad \nabla \cdot \mathbf{B}=0 .
$$

As the curl of electric field $\mathbf{E}$ vanishes, it can be described as the gradient of the electric scalar potential $\phi$

$$
\mathbf{E}=-\nabla \phi
$$

i.e., the magnetic vector potential's contribution to $\mathbf{E}$ in 1.15 is negligible.

\subsubsection{Electric Scalar Potential $\phi$-Formulation}

Using equation (1.58), Maxwell's equations for electroquasistatic fields and the material laws, the following potential equation can be obtained to compute $\phi$

$$
\nabla \cdot \boldsymbol{\sigma} \nabla \phi+\frac{\partial}{\partial t} \nabla \cdot \boldsymbol{\varepsilon} \nabla \phi=0
$$

Eventually, spatial discretisation leads to a system of DAEs

$$
\widetilde{\mathbf{S}} \mathbf{M}_{\sigma} \widetilde{\mathbf{S}}^{\top} \boldsymbol{\Phi}+\widetilde{\mathbf{S}} \mathbf{M}_{\varepsilon} \widetilde{\mathbf{S}}^{\top} \frac{\mathrm{d}}{\mathrm{d} t} \boldsymbol{\Phi}=0
$$

where $\boldsymbol{\Phi}$ contains the degrees of freedom of our problem, i.e. the electric scalar potential on the nodes of the primal grid. However, if boundary conditions are properly set, then one can show

Theorem 5. The system 1.59) under Assumptions 12 and 4 is an ODE.

Theorem 5 follows immediately from Lemma 2 . 


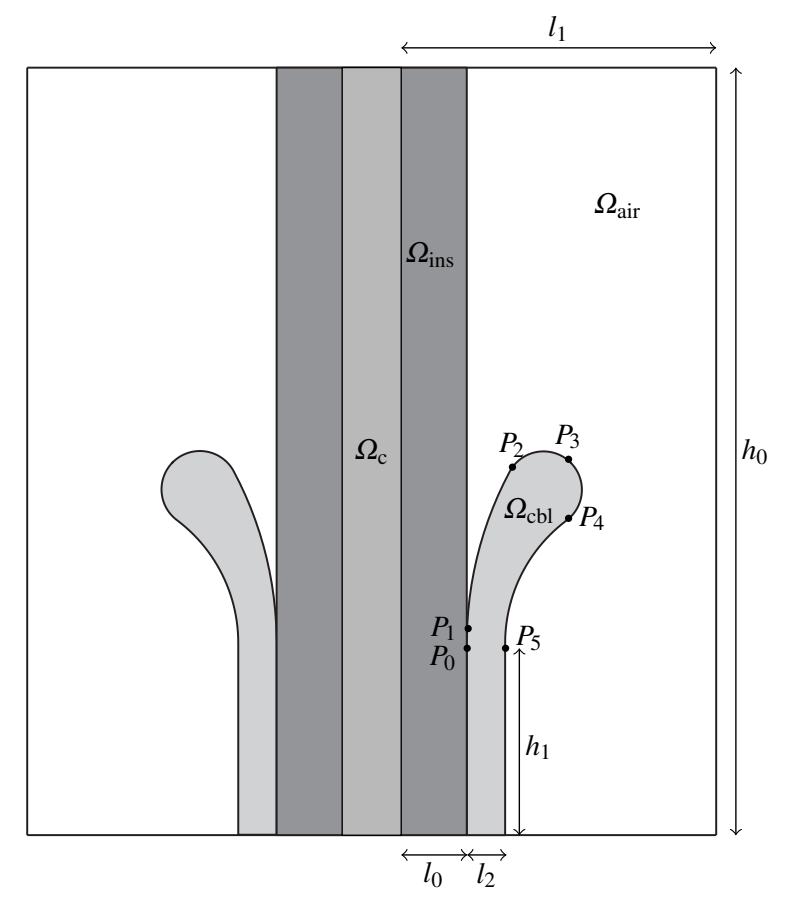

Fig. 1.12 Sketch of electroquasistatic benchmark domain. The distances are $l_{0}=1.2 \cdot 10^{-2} \mathrm{~m}$, $l_{1}=2.5 \cdot 10^{-2} \mathrm{~m}, l_{2}=2 \cdot 10^{-3} \mathrm{~m}, h_{0}=4 \cdot 10^{-2} \mathrm{~m}$ and $h_{1}=1 \cdot 10^{-2} \mathrm{~m}$. To describe the arc segments, points $P_{0}=(12,10), P_{1}=(12.03,11), P_{2}=(14.24,18.94), P_{3}=(17.3,19.52), P_{4}=(17.2,16,4)$ and $P_{5}=(14,10)$ are defined. The first arc segment from point $P_{2}$ to $P_{1}$ has an angle of $25.06^{\circ}$ and is described by straight segments with a grid spacing of $3.02^{\circ}$. Both arc segments from $P_{3}$ to $P_{2}$ as well as from $P_{4}$ to $P_{3}$ have $102.53^{\circ}$ and $28.65^{\circ}$ spacing. The last one from $P_{4}$ to $P_{5}$ has $53.13^{\circ}$ and $7.16^{\circ}$ spacing.

Benchmark 3 In a DC high-voltage cable, the insulation between the inner highvoltage electrode and the outer shielding layer carries a large electric field strength. At the end of the cable $\left(\Omega_{\mathrm{cbl}}\right)$, the voltage has to drop along the surface of the insulation layer $\left(\Omega_{\mathrm{ins}}\right)$ with a substantially smaller electric field strength. This necessitates the design of a so-called cable termination with field-shaping capability. A sketch of the domain and its distances can be seen in Fig. 1.12

The computational domain is $\Omega=\Omega_{\mathrm{air}} \cup \Omega_{\mathrm{ins}}$ and the rest of the domain is modelled via boundary conditions and thus not considered by the discretisation. In the air region $\Omega_{\mathrm{air}}$ the conductivity $\sigma$ is set to zero and the permittivity of vacuum $\varepsilon_{0}=8.85 \cdot 10^{-12} \mathrm{~F} / \mathrm{m}$ is assumed. The insulating domain $\Omega_{\mathrm{ins}}$ has conductivity $1 \mathrm{~S} / \mathrm{m}$ and permittivity $6 \varepsilon_{0}$.

Due to symmetry reasons, only an axisymmetric cross-section (i.e. the right half of the domain sketched in Fig. 1.12 is simulated. The cable endings $\Omega_{\mathrm{cbl}}$ are modelled by zero Dirichlet boundary conditions $(e b c)$ on the boundary $\Gamma_{\mathrm{cbl}}=$ $\partial \Omega_{\mathrm{cbl}} \cap\left(\bar{\Omega}_{\mathrm{ins}} \cup \bar{\Omega}_{\mathrm{air}}\right)$. Similarly, the domain $\Omega_{\mathrm{c}}$ is modelled by non-homogeneous 
Dirichlet boundary conditions that set the potential $\phi$ to a time dependent value $f(t)$ on $\Gamma_{\mathrm{c}}=\partial \Omega_{\mathrm{ins}} \cap \bar{\Omega}_{\mathrm{c}}$, see Fig. 1.13 a. At the rest of the boundary zero Neumann boundary conditions $(\mathrm{mbc})$ are set.

Using the Finite Element Method yields 2892 number of nodes and 2078 degrees of freedom. Time integration is carried out with the implicit Euler method from time $t_{0}=0 \mathrm{~s}$ to $t_{\mathrm{end}}=2 \cdot 10^{-3} \mathrm{~s}$ with step size $\Delta t=1 \cdot 10^{-5} \mathrm{~s}$. The steady state solution is set as initial condition. Fig. 1.13 shows the excitation function $f(t)$ and the electric energy $E_{\mathrm{elec}}=\frac{1}{2} \int_{\Omega} \mathbf{E} \cdot \mathbf{D} \mathrm{d} \Omega \approx \frac{1}{2} \widehat{\mathbf{e}}^{\top} \mathbf{M}_{\varepsilon} \widehat{\mathbf{e}}$ over time.

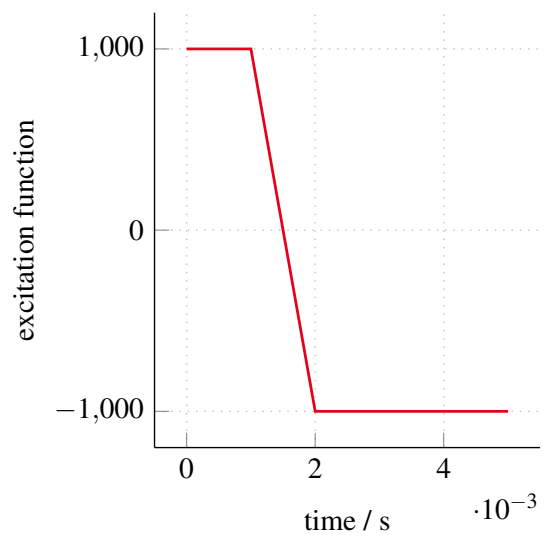

(a) Excitation function $\mathrm{f}(\mathrm{t})$.

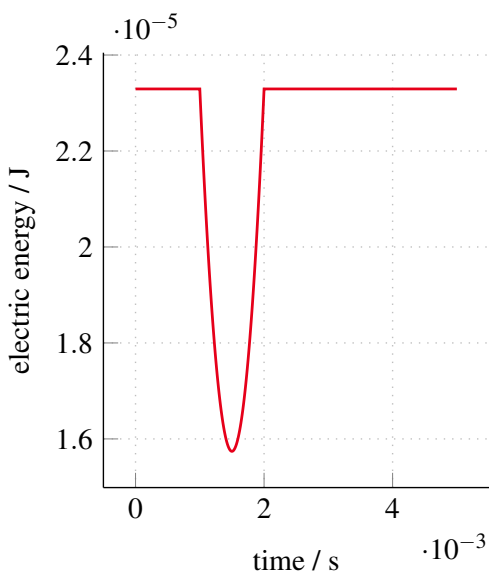

(b) Electric energy.

Fig. 1.13 Electric energy and excitation function of benchmark 3

For the electroquasistatic problem other formulations are not common as (1.59) has convenient properties, e.g. a low-number of degrees of freedom, since no vectorial fields are needed and ordinary differential character. Rarely, a mixed charge/potential formulation [74]

$$
\begin{aligned}
\widetilde{\mathbf{S}} \mathbf{M}_{\sigma} \widetilde{\mathbf{S}}^{\top} \boldsymbol{\Phi}+\frac{\mathrm{d}}{\mathrm{d} t} \mathbf{q} & =0, \\
\mathbf{q}-\widetilde{\mathbf{S}} \mathbf{M}_{\mathcal{\varepsilon}} \widetilde{\mathbf{S}}^{\top} \boldsymbol{\Phi} & =0 .
\end{aligned}
$$

is employed which is a simple and easy to solve DAE index-1 system.

\subsubsection{Magnetoquasistatic Maxwell's Equations}

Now the magnetoquasistatic case is studied. Following Definition $1 \mathrm{c}$ ) Maxwell's equations take the form 


$$
\nabla \times \mathbf{E}=-\frac{\partial \mathbf{B}}{\partial t}, \quad \nabla \times \mathbf{H}=\mathbf{J}, \quad \nabla \cdot \mathbf{D}=\rho, \quad \nabla \cdot \mathbf{B}=0 .
$$

Due to the non-vanishing curl of the electric field strength, a scalar potential formulation is no longer possible. Several competing vector potential formulations are common, see e.g. [25].

\subsubsection{Magnetic Vector Potential A-Formulations}

Using the definition of the magnetic vector and the electric scalar potentials $\mathbf{A}$ and $\phi$ (see equation 1.15) and inserting the material laws and magnetoquasistatic equations into each other, one finds the curl-curl equation

$$
\boldsymbol{\sigma}\left(\frac{\partial \mathbf{A}}{\partial t}+\nabla \phi\right)+\nabla \times(\boldsymbol{v} \nabla \times \mathbf{A})=\mathbf{J}_{\mathrm{s}}
$$

In a three dimensional domain, a gauge condition is necessary to ensure uniqueness of solution, due to the kernel of the curl-operator $\left(\mathbf{C S}^{\top}=0\right)$. The so-called $\mathrm{A}^{*}$ formulation exploits this freedom of choice and assumes that the gradient of the electric scalar potential is zero $(\nabla \phi=0)$. Applying it yields the spatially discretised magnetoquasistatic curl-curl equation

$$
\mathbf{M}_{\sigma} \frac{\mathrm{d}}{\mathrm{d} t} \widehat{\mathbf{a}}+\mathbf{C}^{\top} \mathbf{M}_{v} \mathbf{C} \widehat{\mathbf{a}}=\widehat{\mathbf{j}}_{\mathrm{s}}
$$

with $\widehat{\mathbf{j}}_{\mathrm{s}}$ known and $\widehat{\mathbf{a}}$ containing the degrees of freedom (the magnetic vector potential integrated on the edges of the primal grid). Alternatively, mixed formulations incorporating a gauging conditions have been proposed, e.g. [28]

$$
\left(\begin{array}{cc}
\mathbf{M}_{\sigma} & 0 \\
0 & 0
\end{array}\right) \frac{\mathrm{d}}{\mathrm{d} t}\left(\begin{array}{c}
\widehat{\mathbf{a}} \\
\boldsymbol{\Phi}
\end{array}\right)+\left(\begin{array}{ccc}
\mathbf{C}^{\top} \mathbf{M}_{v} \mathbf{C} & \mathbf{M}_{1} \widetilde{\mathbf{S}}^{\top} \\
-\widetilde{\mathbf{S}} \mathbf{M}_{1} & -\mathbf{M}_{\mathrm{N}}^{-1}
\end{array}\right)\left(\begin{array}{c}
\widehat{\mathbf{a}} \\
\boldsymbol{\Phi}
\end{array}\right)=\left(\begin{array}{c}
\widehat{\mathbf{j}}_{\mathrm{s}} \\
0
\end{array}\right)
$$

where $\mathbf{M}_{1}$ is a regularised version of $\mathbf{M}_{\sigma}$ and $\mathbf{M}_{\mathrm{N}}$ is a regular matrix to ensure the correct physical units as in (1.42). Using the Schur complement, one derives a grad-div regularisation, e.g. [31, where

$$
\mathbf{Z}_{\sigma}=\mathbf{M}_{1} \widetilde{\mathbf{S}}^{\top} \mathbf{M}_{\mathrm{N}} \widetilde{\mathbf{S}} \mathbf{M}_{1}
$$

can be used to finally arrive at

Assumption 5 Let us assume that system (1.60) is rewritten as

$$
\mathbf{M}_{\sigma} \frac{\mathrm{d}}{\mathrm{d} t} \widehat{\mathbf{a}}+\mathbf{K}_{v} \widehat{\mathbf{a}}=\widehat{\mathbf{j}}_{\mathrm{s}},
$$

where $\mathbf{K}_{v}=\mathbf{C}^{\top} \mathbf{M}_{v} \mathbf{C}+\mathbf{Z}_{\sigma}$, provided $\mathbf{Z}_{\sigma}$ is a positive semidefinite matrix that enforces the matrix pencil $\lambda \mathbf{M}_{\sigma}+\mathbf{K}_{v}$ to be positive definite for $\lambda>0$. 
Theorem 6. Under Assumptions 1, 2, 4 and 5, system (1.61) has Kroenecker and tractability index 1 .

The proof of the Kronecker index of system (1.61) has been originally given in [71]. More recently, [57] obtained the same result for the tractability index and [7] used the tractability index concept to analyse the DAE index of this formulation with an attached network description.

Instead of using $\mathbf{Z}_{\sigma}$, various gauging techniques have been proposed for this formulation, such as the tree-cotree gauge [68] or the weak gauging property of iterative linear solvers [30]. Due to the simple structure of $\mathbf{M}_{\sigma}$ and as long as the gauge leads to a positive definite matrix pencil, the index can be derived analogously as before.

Benchmark 4 A common benchmark for magnetoquasistatic models are inductors with a metal core. The example in Fig. 1.14 from [33] features an aluminium core, i.e. $\sigma=35 \cdot 10^{6} \mathrm{~S} / \mathrm{m}$ in $\Omega_{c}$ with a copper coil $\Omega_{\mathrm{str}}$ surrounded by air $\Omega_{\text {air. }}$. The coil is given by the stranded conductor model consisting of 120 turns with conductivity $\sigma=1 \cdot 10^{6} \mathrm{~S} / \mathrm{m}$. The conductivity in $\Omega_{\mathrm{air}}$ is zero and disregarded in $\Omega_{\mathrm{str}}$ in the construction of $\mathbf{M}_{\sigma}$ as eddy currents are assumed negligible in the windings. Vacuum permeability $\mu=4 \pi \cdot 1 \cdot 10^{-7} \mathrm{H} / \mathrm{m}$ is assumed everywhere $(\Omega)$ and electric boundary conditions are enforced $\Gamma=\Gamma_{e b c}$, $c f$. (1.4). The FIT discretisation uses an equidistant hexahedral grid with step size $10^{-3}$, which leads to 3528 elements.

The discretisation of the winding function for the $A^{*}$ formulation can be visualised in Fig. 1.15 As no gauging is performed, 9958 degrees of freedom arise.

For the simulation, the source current density is $\widehat{\mathbf{j}}_{\mathrm{s}}=\mathbf{X}_{\mathrm{s}} \mathbf{i}_{\mathrm{s}}$, where $\mathbf{X}_{\mathrm{s}}$ is the discretisation of the winding function in Fig. 1.15 , $\mathbf{i}_{\mathrm{s}}=\sin \left(2 \pi f_{\mathrm{s}} t\right)$ is the source current and the frequency $f_{\mathrm{s}}$ is $500 \mathrm{~Hz}$. Time integration is performed with the implicit Euler method with step size $\Delta t=2 \cdot 10^{-5} \mathrm{~s}$ from time $t_{0}=0 \mathrm{~s}$ to $t_{\mathrm{end}}=2 \cdot 10^{-3} \mathrm{~s}$ and zero initial condition. Fig. 1.16 shows the magnetic energy $E_{\mathrm{mag}}=\frac{1}{2} \int_{\Omega} \mathbf{H} \cdot \mathbf{B} \mathrm{d} \Omega \approx$ $\frac{1}{2} \widehat{\mathbf{b}}^{\top} \mathbf{M}_{v} \widehat{\mathbf{b}}$ over time.

\subsubsection{Electric Vector Potential T- $\Omega$-Formulation}

Maxwell's equations and their material laws in magnetoquasistatics can also be expressed in the T- $\Omega$ formulation. Using the electric vector and magnetic scalar potentials from equation $(1.16)$, the system reads

$$
\begin{aligned}
\nabla \times(\boldsymbol{\rho} \nabla \times \mathbf{T})+\boldsymbol{\mu} \frac{\partial \mathbf{T}}{\partial t}-\boldsymbol{\mu} \nabla \frac{\partial \psi}{\partial t} & =-\boldsymbol{\mu} \frac{\partial \mathbf{H}_{\mathrm{s}}}{\partial t}-\nabla \times \mathbf{E}_{\mathrm{s}} \\
\nabla \cdot(\boldsymbol{\mu} \mathbf{T})-\nabla \cdot(\boldsymbol{\mu} \nabla \psi) & =-\nabla \cdot\left(\boldsymbol{\mu} \mathbf{H}_{\mathrm{s}}\right),
\end{aligned}
$$

with $\mathbf{E}_{\mathrm{s}}$ being the source electric field strength when solid conductors are present. Here, $\boldsymbol{\rho}$ is the electrical resistivity, which corresponds to the inverse of $\boldsymbol{\sigma}$ wherever $\boldsymbol{\sigma} \neq 0$. As in the case of the A- $\phi$ formulation, the gauge condition is set for the 


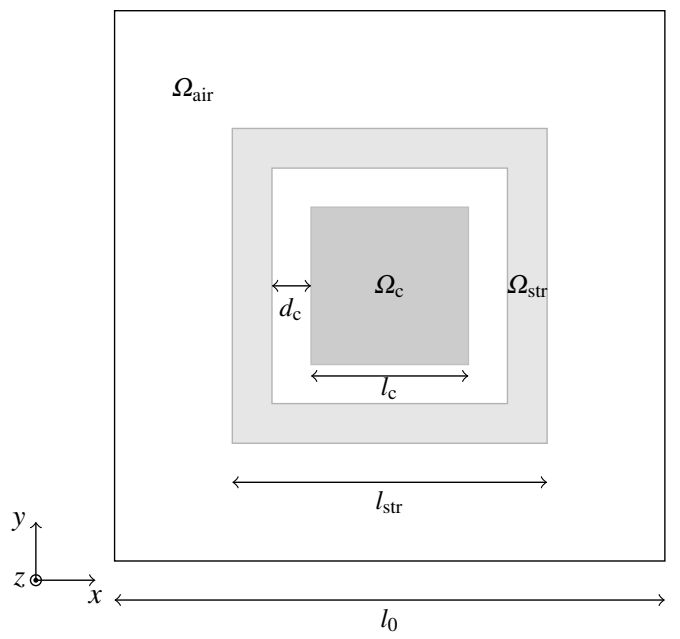

(a) Cross-section of inductor at the $\mathrm{z}=0$ plane.

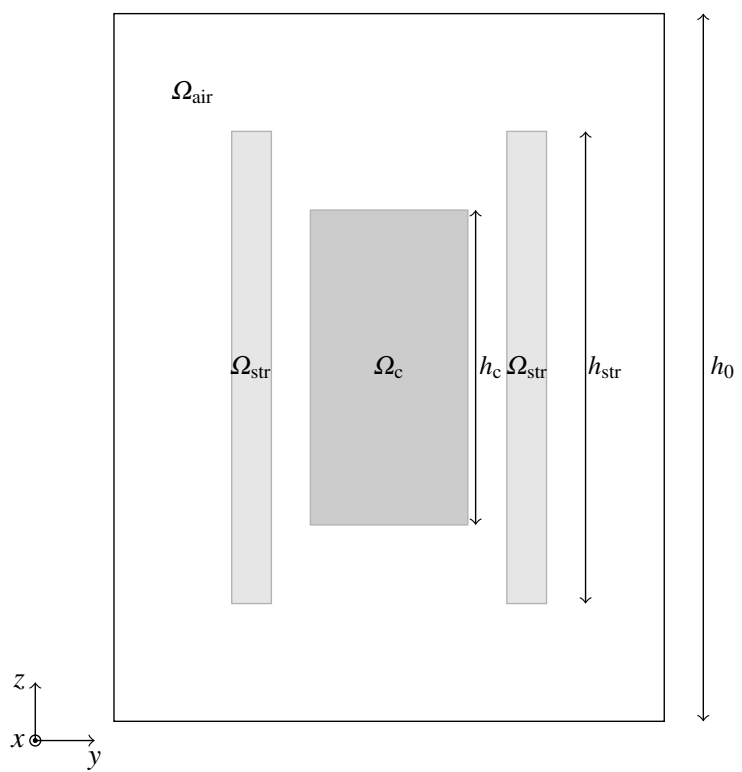

(b) Cross-section of inductor at the $\mathrm{x}=0$ plane.

Fig. 1.14 Magnetoquasistatic model of an inductor with metal core. The distances are $l_{0}=1.4$. $10^{-2} \mathrm{~m}, l_{\mathrm{str}}=8 \cdot 10^{-3} \mathrm{~m} l_{\mathrm{c}}=4 \cdot 10^{-3} \mathrm{~m}, d_{\mathrm{c}}=1 \cdot 10^{-3} \mathrm{~m}, h_{0}=1.8 \cdot 10^{-2} \mathrm{~m}, l_{\mathrm{str}}=1.2 \cdot 10^{-2} \mathrm{~m}$ and $l_{\mathrm{c}}=8 \cdot 10^{-3} \mathrm{~m}$.

spatially discretised version of our system 


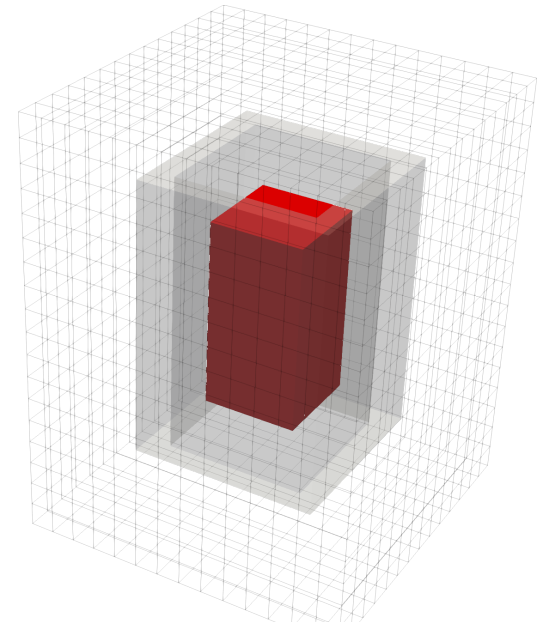

(a) Iron core (red) with surrounding coil (transparent grey)

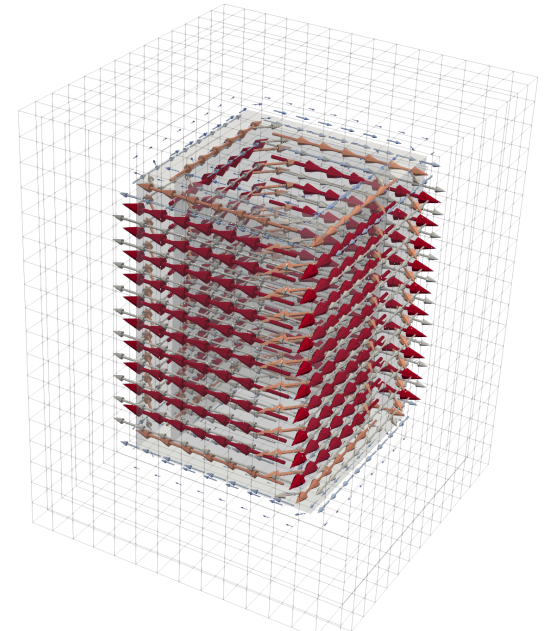

(b) Source current density given by winding function $\mathbf{J}_{\mathrm{s}}=\boldsymbol{\chi}_{\mathrm{s}} i_{\mathrm{s}}$

Fig. 1.15 Simple magnetoquasistatic model of an inductor with metal core. Coil is given by the stranded conductor model (Benchmark 4)

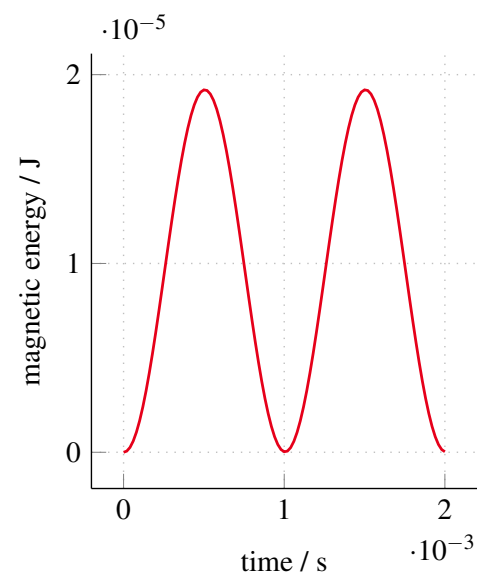

Fig. 1.16 Magnetic energy of the inductor in Fig. 1.14 simulated with the A* formulation.

$$
\begin{aligned}
& \mathbf{C}^{\top} \mathbf{M}_{\rho} \mathbf{C} \widehat{\mathbf{t}}+\mathbf{M}_{\mu} \frac{\mathrm{d}}{\mathrm{d} t} \widehat{\mathbf{t}}+\mathbf{M}_{\mu} \widetilde{\mathbf{S}}^{\top} \frac{\mathrm{d}}{\mathrm{d} t} \Psi=-\mathbf{M}_{\mu} \frac{\mathrm{d}}{\mathrm{d} t} \widehat{\mathbf{h}}_{\mathrm{s}}-\mathbf{C} \widehat{\mathbf{e}}_{\mathrm{s}} \\
& \widetilde{\mathbf{S}} \mathbf{M}_{\mu} \widehat{\mathbf{t}}+\widetilde{\mathbf{S}} \mathbf{M}_{\mu} \widetilde{\mathbf{S}}^{\top} \Psi=-\widetilde{\mathbf{S}} \mathbf{M}_{\mu} \widehat{\mathbf{h}}_{\mathrm{s}},
\end{aligned}
$$

with degrees of freedom $\widehat{t}$, which contains the on the dual grid's edges integrated electric vector potential and $\Psi$, which consists of the magnetic scalar potential on the dual grid's nodes. 
Definition 4. For gauging, a tree $T$ is generated on the dual grid's edges inside the conducting region $\Omega_{\mathrm{c}}$. We define a projector $\tilde{\mathbf{P}}_{\mathrm{t}}$ onto the cotree of $T$ and truncate it by deleting all the linearly dependent columns to obtain $\mathbf{P}_{\mathrm{t}}$. As gauging condition we set $\mathbf{Q}_{\mathbf{t}} \widehat{\mathbf{t}}=0$, where $\mathbf{Q}_{\mathrm{t}}$ spans the kernel of $\mathbf{P}_{\mathrm{t}}^{\top}$. This corresponds to setting to zero the values of the electric vector potential on the edges $T$.

$$
\begin{aligned}
\mathbf{P}_{\mathrm{t}}^{\top} \mathbf{C}^{\top} \mathbf{M}_{\rho} \mathbf{C} \mathbf{P}_{\mathrm{t}} \widehat{\mathbf{t}}+\mathbf{P}_{\mathrm{t}}^{\top} \mathbf{M}_{\mu} \mathbf{P}_{\mathrm{t}} \frac{\mathrm{d}}{\mathrm{d} t} \widetilde{\mathbf{t}}+\mathbf{P}_{\mathrm{t}}^{\top} \mathbf{M}_{\mu} \widetilde{\mathbf{S}}^{\top} \frac{\mathrm{d}}{\mathrm{d} t} \psi=-\mathbf{P}_{\mathrm{t}}^{\top} \mathbf{M}_{\mu} \frac{\mathrm{d}}{\mathrm{d} t} \widehat{\mathbf{h}}_{\mathrm{s}}-\mathbf{P}_{\mathrm{t}}^{\top} \mathbf{C} \widehat{\mathbf{e}}_{\mathrm{s}} \\
\widetilde{\mathbf{S}} \mathbf{M}_{\mu} \mathbf{P}_{\mathrm{t}} \widehat{\mathbf{t}}+\widetilde{\mathbf{S}} \mathbf{M}_{\mu} \widetilde{\mathbf{S}}^{\top} \psi=-\widetilde{\mathbf{S}} \mathbf{M}_{\mu} \widehat{\mathbf{h}}_{\mathrm{s}} .
\end{aligned}
$$

Property 1. The matrix $\mathbf{P}_{\mathrm{t}}$ fulfils

1. $\operatorname{det}\left(\mathbf{P}_{\mathrm{t}}^{\top} \mathbf{C}^{\top} \mathbf{M}_{\rho} \mathbf{C P}_{\mathrm{t}}\right) \neq 0$,

2. $\operatorname{im} \mathbf{P}_{\mathrm{t}} \cap \operatorname{im} \widetilde{\mathbf{S}}^{\top}=\emptyset$.

Property 1.1 is a consequence of the tree-cotree gauge (see [68]) and Property 1.2 follows from Property 1.1 and the fact that $\operatorname{im} \widetilde{\mathbf{S}}^{\top} \subseteq \operatorname{ker} \mathbf{C}($ Lemma 1 ).

Property 2 ([33] ). Every $\mathbf{x} \in \mathbb{R}^{n}$ can be written as $\mathbf{x}=\mathbf{M}_{\mu}^{1 / 2} \widetilde{\mathbf{S}}^{\top} \mathbf{x}_{1}+\mathbf{M}_{\mu}^{-1 / 2} \mathbf{W}^{\top} \mathbf{x}_{2}$, where $n:=\operatorname{rank} \mathbf{M}_{\mu}$ and $\mathbf{W}$ is the matrix whose columns span $\operatorname{ker} \widetilde{\mathbf{S}}$.

Proof. As $\mathbf{M}_{\mu}$ has full rank and is symmetric, $\operatorname{rank}\left(\mathbf{M}_{\mu}^{1 / 2} \widetilde{\mathbf{S}}^{\top}\right)=\operatorname{rank} \widetilde{\mathbf{S}}^{\top}$ and $\operatorname{rank}\left(\mathbf{M}_{\mu}^{-1 / 2} \mathbf{W}^{\top}\right)=\operatorname{rank} \mathbf{W}^{\top}$. Using the rank-nullity theorem together with the fact that both subspaces are orthogonal and thus linear independent, we obtain that their direct sum spans $\mathbb{R}^{n}$.

Theorem 7 ([33]). Under Assumptions 1,2 and 4 the system of DAEs (1.62)-(1.63) has differential index-1.

Proof. As the system has an algebraic constraint, it has at least index-1. Equation 1.63 is differentiated and $\frac{\mathrm{d}}{\mathrm{d} t} \Psi$ is extracted as

$$
\frac{\mathrm{d}}{\mathrm{d} t} \boldsymbol{\Psi}=-\left(\widetilde{\mathbf{S}} \mathbf{M}_{\mu} \widetilde{\mathbf{S}}^{\top}\right)^{-1} \widetilde{\mathbf{S}} \mathbf{M}_{\mu} \mathbf{P}_{\mathrm{t}} \frac{\mathrm{d}}{\mathrm{d} t} \mathbf{t}-\left(\widetilde{\mathbf{S}} \mathbf{M}_{\mu} \widetilde{\mathbf{S}}^{\top}\right)^{-1} \widetilde{\mathbf{S}} \mathbf{M}_{\mu} \widehat{\mathbf{h}}_{\mathbf{s}} .
$$

This can be inserted into equation 1.62 and now it is sufficient to see that $\operatorname{det}\left(\mathbf{P}_{\mathrm{t}} \mathbf{Z} \mathbf{P}_{\mathrm{t}}\right) \neq 0$ for

$$
\mathbf{Z}=\left(\mathbf{M}_{\mu}-\mathbf{M}_{\mu} \widetilde{\mathbf{S}}^{\top}\left(\widetilde{\mathbf{S}} \mathbf{M}_{\mu} \widetilde{\mathbf{S}}^{\top}\right)^{-1} \widetilde{\mathbf{S}} \mathbf{M}_{\mu}\right) .
$$

We can write $\mathbf{M}_{\mu}^{1 / 2} \mathbf{P}_{\mathrm{t}} \mathbf{x}=\mathbf{M}_{\mu}^{1 / 2} \widetilde{\mathbf{S}}^{\top} \mathbf{x}_{1}+\mathbf{M}_{\mu}^{-1 / 2} \mathbf{W}^{\top} \mathbf{x}_{2}$ (Property2). As $\mathbf{M}_{\mu}^{1 / 2}$ is invertible, $\mathbf{P}_{\mathrm{t}} \mathbf{x}=\widetilde{\mathbf{S}}^{\top} \mathbf{x}_{1}+\mathbf{M}_{\mu}^{-1} \mathbf{W}^{\top} \mathbf{x}_{2}$ and, as $\mathbf{P}_{\mathrm{t}} \mathbf{x} \neq \widetilde{\mathbf{S}}^{\top} \mathbf{x}_{1}$ (Property 1 ), $\mathbf{M}_{\mu}^{-1} \mathbf{W}^{\top} \mathbf{x}_{2} \neq 0$. Thus

$$
\mathbf{x}^{\top} \mathbf{P}_{\mathrm{t}}^{\top} \mathbf{Z} \mathbf{P}_{\mathrm{t}} \mathbf{x}=\mathbf{x}_{2}^{\top} \mathbf{W} \mathbf{M}_{\mu}^{-1} \mathbf{W}^{\top} \mathbf{x}_{2}>0
$$

as long as $\mathbf{x} \neq 0$. We conclude that $\mathbf{P}_{\mathrm{t}}^{\top} \mathbf{Z} \mathbf{P}_{\mathrm{t}}$ is positive definite. 


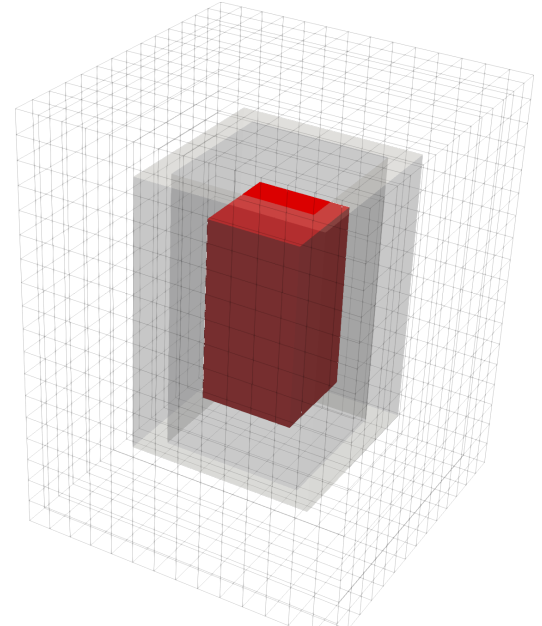

(a) Iron core (red) with surrounding coil (transparent grey)

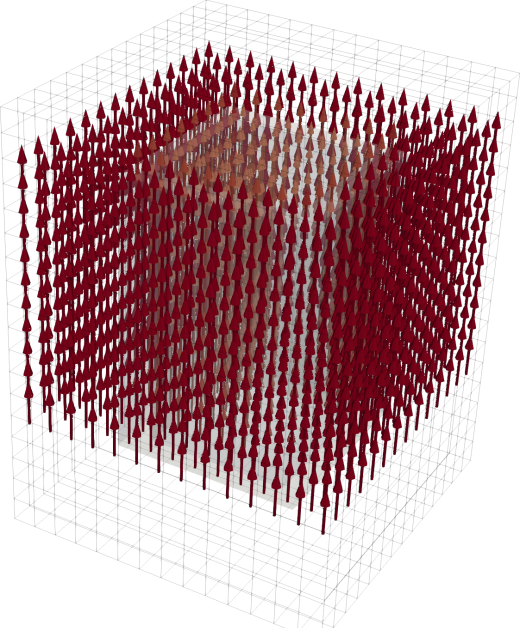

(b) Source magnetic field strength $\mathbf{H}_{\mathrm{s}}$ such that $\nabla \times \mathbf{H}_{\mathrm{s}}=\boldsymbol{\chi}_{\mathrm{s}} i_{\mathrm{s}}$

Fig. 1.17 Simple magnetoquasistatic model of an inductor with metal core. Coil is given by the stranded conductor model (Benchmark 5)

Benchmark 5 The physical specifications and discretisation of the benchmark example of the $T-\Omega$ formulation is equivalent to Benchmark 4.

The construction of the winding function is different and it is depicted in Fig. 1.17 This time a tree-cotree gauge is performed, which yield only 4610 degrees of freedom.

Again for the simulation the source current is set to $\mathbf{i}_{\mathrm{s}}=\sin \left(2 \pi f_{\mathrm{s}} t\right)$ with frequency $f_{\mathrm{s}}=500 \mathrm{~Hz}$. The source magnetic field is $\widehat{\mathbf{h}}_{\mathrm{s}}=\mathbf{Y}_{\mathrm{s}} \mathbf{i}_{\mathrm{s}}$, with $\mathbf{Y}_{\mathrm{s}}$ being the discretisation of the winding function in Fig. 1.17 Like in Benchmark 4 time integration is performed with implicit Euler with step size $\Delta t=2 \cdot 10^{-5} \mathrm{~s}$ from time $t_{0}=0 \mathrm{~s}$ to $t_{\mathrm{end}}=2 \cdot 10^{-3} \mathrm{~s}$ and with zero initial condition. The resulting magnetic energy is depicted in Figure 1.18 .

Remark 8. Note that the magnetic energy obtained with the $\mathrm{A}^{*}$ formulation in Figure 1.16 and the one obtained with the T- $\Omega$ one in Figure 1.18 differ. Both formulations are dual to each other, i.e., their degrees of freedom are on dual sides of Maxwell's House in Figure 1.5. They converge to the unique physical solution from below and above. This property can be used in order to study the error of the spatial discretisation (see [1]). 


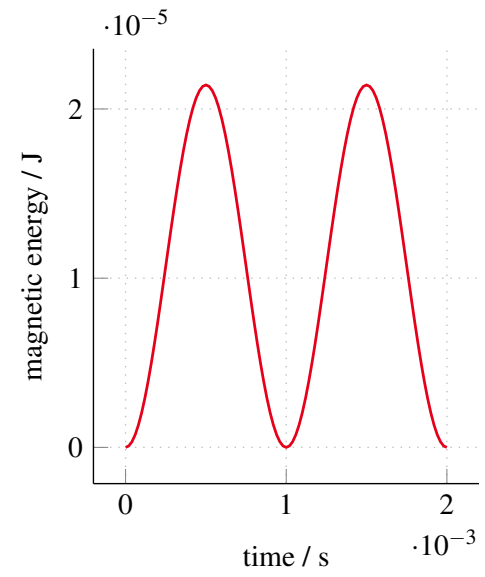

Fig. 1.18 Magnetic energy of the inductor in Fig. 1.14 simulated with the T- $\Omega$ formulation.

\subsubsection{Darwin Model}

In many situations, phenomena related to electric energy, magnetic energy and Joule losses coincide, while at the same time, for the considered operating frequencies, the wave lengths are much larger than the model size, which indicates that wave propagation effects can be neglected. An example thereof is a filter design with concentrated elements, i.e., coils and capacitors connected by strip lines, arranged on a printed circuit board [84]. In such models, resonances between the discrete elements and Joule losses in all conducting parts need to be considered, whereas for the considered frequency range, cross-talk due to electromagnetic radiation is irrelevant. For such configurations, a combination of electroquasistatics and magnetoquasistatics in the form of the Darwin approximation of the Maxwell equations is the appropriate formulation.

The Darwin approximation starts from the decomposition of the electric field strength $\mathbf{E}=\mathbf{E}_{\text {irr }}+\mathbf{E}_{\text {rem }}$ into an irrotational part $\mathbf{E}_{\text {irr }}$ and a remainder part $\mathbf{E}_{\text {rem }}$. This decomposition is not unique, in contrast to the Helmholtz decomposition, which can be considered as a special case enforcing $\mathbf{E}_{\text {rem }}$ to be solenoidal. In this paper, the non-uniqueness of the decomposition will be resolved when choosing a gauge condition below. The Darwin approximation consists of removing the displacement currents related to $\mathbf{E}_{\text {rem }}$ from the law of Ampère-Maxwell. This affects the set of Maxwell equations in the sense that, in the formulations derived below, second derivatives with respect to time vanish and the overall PDE looses its hyperbolic character, which is equivalent to neglecting wave propagation effects. The set of relevant equations is 


$$
\begin{aligned}
\nabla \times \mathbf{E}_{\mathrm{rem}} & =-\frac{\partial \mathbf{B}}{\partial t} \quad ; \\
\nabla \times(\boldsymbol{v B}) & =\mathbf{J}_{\mathrm{s}}+\boldsymbol{\sigma} \mathbf{E}_{\mathrm{irr}}+\boldsymbol{\sigma} \mathbf{E}_{\mathrm{rem}}+\frac{\partial}{\partial t}\left(\varepsilon \mathbf{E}_{\mathrm{irr}}\right) \quad ; \\
\nabla \cdot \mathbf{B} & =0 ; \\
\nabla \cdot\left(\varepsilon \mathbf{E}_{\mathrm{irr}}\right)+\nabla \cdot\left(\varepsilon \mathbf{E}_{\mathrm{rem}}\right) & =\rho .
\end{aligned}
$$

Almost all publications on the Darwin approximation are limited to the case with homogeneous materials. They choose the Helmholtz decomposition, i.e., $\nabla \cdot \mathbf{E}_{\mathrm{rem}}=$ 0 , which causes the term $\nabla \cdot\left(\varepsilon \mathbf{E}_{\mathrm{rem}}\right)$ in $(1.67)$ to vanish [39, 61]. In the more general case considered here, however, this term is important as it models the charges accumulating at material interfaces due to the induced electric field strength [59].

An ambiguity arises when expressing the continuity equation $\nabla \cdot \mathbf{J}+\frac{\partial \rho}{\partial t}=0$. When inserting Gauss law, the result reads

$$
\nabla \cdot \mathbf{J}_{\mathrm{s}}+\nabla \cdot\left(\boldsymbol{\sigma} \mathbf{E}_{\mathrm{irr}}\right)+\nabla \cdot\left(\boldsymbol{\sigma} \mathbf{E}_{\mathrm{rem}}\right)+\frac{\partial}{\partial t}\left(\nabla \cdot \varepsilon \mathbf{E}_{\mathrm{irr}}\right)+\frac{\partial}{\partial t}\left(\nabla \cdot \varepsilon \mathbf{E}_{\mathrm{rem}}\right)=0,
$$

whereas when applying applying the divergence operator to (1.65), the last term in (1.68) is missing. Hence, the Darwin model introduces an anomaly in the continuity equation, which can only be alleviated by also neglecting the displacement currents due to $\mathbf{E}_{\text {rem }}$ in the continuity equation.

\subsubsection{1 $\phi-A-\psi$ formulation of the Darwin model}

The irrotational part in of the electric field strength is represented by an electric scalar potential $\phi$, i.e., $\mathbf{E}_{\text {irr }}=-\nabla \phi$. The magnetic Gauss law (1.66) is resolved by the definition of the magnetic vector potential $\mathbf{A}$, i.e., $\mathbf{B}=\nabla \times \mathbf{A}$, whereas Faraday's law (1.64) is fulfilled by the definition of an additional scalar potential $\psi$ such that $\mathbf{E}_{\text {rem }}=-\frac{\partial \mathbf{A}}{\partial t}-\nabla \psi$. The law of Ampère-Darwin, Gauss' law and the continuity equation become

$$
\begin{array}{r}
\nabla \times(\boldsymbol{v} \nabla \times \mathbf{A})+\boldsymbol{\sigma} \frac{\partial \mathbf{A}}{\partial t}+\boldsymbol{\sigma} \nabla \psi+\boldsymbol{\sigma} \nabla \phi+\varepsilon \nabla \frac{\partial \phi}{\partial t}=\mathbf{J}_{\mathrm{s}} \\
-\nabla \cdot\left(\varepsilon \frac{\partial \mathbf{A}}{\partial t}\right)-\nabla \cdot(\varepsilon \nabla \psi)-\nabla \cdot(\varepsilon \nabla \phi)=\rho \\
-\nabla \cdot\left(\boldsymbol{\sigma} \frac{\partial \mathbf{A}}{\partial t}\right)-\nabla \cdot(\boldsymbol{\sigma} \nabla \psi)-\nabla \cdot(\boldsymbol{\sigma} \nabla \phi)-\nabla \cdot\left(\varepsilon \nabla \frac{\partial \phi}{\partial t}\right)=0
\end{array}
$$

The introduced potentials $\phi, \mathbf{A}$ and $\psi$ lead to too many degrees of freedom. The electric field strength is decomposed as 


$$
\mathbf{E}=\underbrace{\overbrace{-\nabla \phi}^{\mathbf{E}_{\phi}}}_{\mathbf{E}_{\text {irr }}} \underbrace{\overbrace{-\frac{\partial \mathbf{A}}{\partial t} \overbrace{-\nabla \psi}^{\mathbf{E}_{\mathbf{A}}}}^{\mathbf{E}_{\psi}}}_{\mathbf{E}_{\mathrm{rem}}}
$$

Irrotational parts of the electric field strength can be attributed to $\mathbf{E}_{\phi}, \mathbf{E}_{\mathbf{A}}$ or $\mathbf{E}_{\psi}$. However, the splitting determines which displacement currents are considered in the law of Ampère-Maxwell and which are only considered in the electric law of Gauss. All components of $\mathbf{E}$ contribute to the Joule losses and contribute in the electric Gauss law, whereas only $\mathbf{E}_{\phi}$ contributes to the displacement currents. The component $\mathbf{E}_{\psi}$ can be fully integrated into $\mathbf{E}_{\mathbf{A}}$.

The choice of $\psi$ determines the fraction of the displacement currents which is neglected in the Darwin model. In the case of a homogeneous material distribution, the choice $\psi=0$ implies a Helmholtz splitting of $\mathbf{E}$ into the irrotational part $\mathbf{E}_{\text {irr }}$ and a solenoidal part $\mathbf{E}_{\text {rem }}$. Then, the Darwin approximation amounts to neglecting the displacement currents related to the solenoidal part of the electric field strength. Other choices for $\psi$ lead to other Darwin models yielding different results. Hence, Darwin's approximation to the Maxwell equations is not gauge-invariant [61].

\subsubsection{2 $\psi-\mathrm{A}^{*}$-formulation of the Darwin model}

A straightforward choice for $\psi$ is $\psi=0$ in which case, it makes sense to combine (1.69) and 1.70), i.e.,

$$
\begin{aligned}
& \nabla \times(\boldsymbol{v} \nabla \times \mathbf{A})+\boldsymbol{\sigma} \frac{\partial \mathbf{A}}{\partial t}+\boldsymbol{\sigma} \nabla \phi+\varepsilon \nabla \frac{\partial \phi}{\partial t}=\mathbf{J}_{\mathrm{s}} \\
&-\nabla \cdot\left(\varepsilon \frac{\partial \mathbf{A}}{\partial t}\right)-\nabla \cdot(\varepsilon \nabla \phi)=\rho
\end{aligned}
$$

The drawback of this formulation is that the charge density must be prescribed, which may be cumbersome in the presence of metallic parts at floating potentials.

\subsubsection{Alternative $\psi$ - $\mathrm{A}^{*}$-formulation of the Darwin model}

Another possible formulation [58] arises with $\psi=0$, but by combining [1.69] and (1.71), i.e.,

$$
\begin{array}{r}
\nabla \times(\boldsymbol{v} \nabla \times \mathbf{A})+\boldsymbol{\sigma} \frac{\partial \mathbf{A}}{\partial t}+\boldsymbol{\sigma} \nabla \phi+\varepsilon \nabla \frac{\partial \phi}{\partial t}=\mathbf{J}_{\mathrm{s}} \\
-\nabla \cdot\left(\boldsymbol{\sigma} \frac{\partial \mathbf{A}}{\partial t}\right)-\nabla \cdot(\boldsymbol{\sigma} \nabla \phi)-\nabla \cdot\left(\varepsilon \nabla \frac{\partial \phi}{\partial t}\right)=0 .
\end{array}
$$

Here, however, the magnetic vector potential $\mathbf{A}$ is not uniquely defined in the nonconductive model parts. Then, a gauge is necessary is fix the irrotational part of $\mathbf{A}$ 


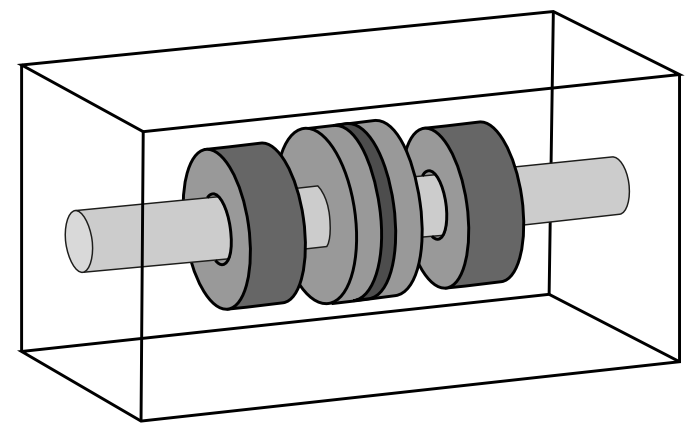

Fig. 1.19 Benchmark example for Darwin model, originally proposed in [59], see Benchmark 6 ]

in the non-conductive model parts. During post-processing, one should discard the irrotational part of $\mathbf{A}$ when calculating the electric field strength or displacement current density in the non-conducting parts.

\subsubsection{Discretisation of the Darwin model}

The discrete counterpart of 1.73 and 1.74 reads

$$
\begin{aligned}
\mathbf{C}^{\top} \mathbf{M}_{v} \mathbf{C} \widehat{\mathbf{a}}+\mathbf{M}_{\sigma} \frac{\mathrm{d}}{\mathrm{d} t} \widehat{\mathbf{a}}-\mathbf{M}_{\sigma} \widetilde{\mathbf{S}}^{\top} \Phi-\mathbf{M}_{\varepsilon} \widetilde{\mathbf{S}}^{\top} \frac{\mathrm{d}}{\mathrm{d} t} \Phi=\widehat{\mathbf{j}}_{\mathrm{s}} ; \\
\widetilde{\mathbf{S}} \mathbf{M}_{\varepsilon} \frac{\mathrm{d}}{\mathrm{d} t} \widehat{\mathbf{a}}+\widetilde{\mathbf{S}} \mathbf{M}_{\varepsilon} \widetilde{\mathbf{S}}^{\top} \Phi=\mathbf{q} ;
\end{aligned}
$$

whereas the discrete counterpart of 1.75 and 1.76 reads

$$
\begin{aligned}
\mathbf{C}^{\top} \mathbf{M}_{v} \mathbf{C} \widehat{\mathbf{a}}+\mathbf{M}_{\sigma} \frac{\mathrm{d}}{\mathrm{d} t} \widehat{\mathbf{a}}-\mathbf{M}_{\sigma} \widetilde{\mathbf{S}}^{\top} \boldsymbol{\Phi}-\mathbf{M}_{\mathcal{E}} \widetilde{\mathbf{S}}^{\top} \frac{\mathrm{d}}{\mathrm{d} t} \boldsymbol{\Phi}=\widehat{\mathbf{j}}_{\mathrm{s}} ; \\
\widetilde{\mathbf{S}} \mathbf{M}_{\sigma} \frac{\mathrm{d}}{\mathrm{d} t} \widehat{\mathbf{a}}+\widetilde{\mathbf{S}} \mathbf{M}_{\sigma} \widetilde{\mathbf{S}}^{\top} \Phi+\widetilde{\mathbf{S}} \mathbf{M}_{\mathcal{\varepsilon}} \widetilde{\mathbf{S}}^{\top} \frac{\mathrm{d}}{\mathrm{d} t} \boldsymbol{\Phi}=0
\end{aligned}
$$

with all matrices defined as above. The second formulation needs a gauge for the non-conducting model parts as previously discussed in Assumption 5 .

In [59], simulation results for the Darwin approximation, the electroquasistatic approximation and the magnetoquasistatic approximation are compared to results obtained by a full-wave solver, serving as a reference. This comparison clearly shows the limitations (the neglected wave propagation effects) and the advantages (more accurate than electroquasistatics and magnetoquasistatics and faster than a full-wave solver) of the Darwin model.

A DAE index analysis of the systems $(1.77)-(1.78)$ and $(1.79)-(1.80)$ is an open research question. Also, the following benchmark is merely a literature reference. 
Benchmark 6 The Darwin benchmark example shown in Fig. 1.19 is taken from [59]. It is axisymmetric and consists of conductive, dielectric and magnetic pieces. A solid conductor is connected to two plates which form a capacitor in combination with the dielectric material layer in between. Two solid ferrite rings surround the conductor. The problem is excited by potentials $\phi_{1}$ and $\phi_{0}$ at the ends of the conductor, the remaining boundaries are mbc. Geometry specifications and material data can be found in [59]. Koch et al. propose to regularise the model by an artificial conductivity.

\subsection{Conclusions}

This paper has discussed various formulation for low and high-frequency problems in computational electromagnetics. In contrast to electric circuit simulation, e.g. [92], most formulations are rather harmless, i.e., they lead to a systems of low DAE index $(\leq 2)$. More precisely, the only index-2 problem arises in the case of considering Maxwell's equations in A- $\phi$-potential formulation with a Coulomb gauge. It has been shown that this can be mitigated by a reformulation based on Lorenz' gauge. Obviously, when coupling various formulations with each other or with electric circuits, the situation becomes more complex.

\section{References}

1. Albanese, R., Coccorese, E., Martone, R., Miano, G., Rubinacci, G.: On the numerical solution of the nonlinear three-dimensional eddy current problem. IEEE Trans. Magn. 27(5), 3990-3995 (1991). DOI 10.1109/20.104976

2. Alonso Rodríguez, A., Raffetto, M.: Unique solvability for electromagnetic boundary value problems in the presence of partly lossy inhomogeneous anisotropic media and mixed boundary conditions. M3AS 13(04), 597-611 (2003). DOI 10.1142/S0218202503002672

3. Alonso Rodríguez, A., Valli, A.: Eddy Current Approximation of Maxwell Equations, Modeling, Simulation and Applications, vol. 4. Springer, Heidelberg (2010). DOI 10.1007/ 978-88-470-1506-7

4. Alotto, P., De Cian, A., Molinari, G.: A time-domain 3-D full-Maxwell solver based on the cell method. IEEE Trans. Magn. 42(4), 799-802 (2006). DOI 10.1109/tmag.2006.871381

5. Assous, F., Ciarlet, P., Labrunie, S.: Mathematical foundations of computational electromagnetism. Springer (2018)

6. Außerhofer, S., Bíró, O., Preis, K.: Discontinuous Galerkin finite elements in time domain eddy-current problems. IEEE Trans. Magn. 45(3), 1300-1303 (2009)

7. Bartel, A., Baumanns, S., Schöps, S.: Structural analysis of electrical circuits including magnetoquasistatic devices. APNUM 61, 1257-1270 (2011). DOI 10.1016/j.apnum.2011.08.004

8. Baumanns, S.: Coupled electromagnetic field/circuit simulation: Modeling and numerical analysis. Ph.D. thesis, Universität zu Köln, Köln (2012)

9. Baumanns, S., Clemens, M., Schöps, S.: Structural aspects of regularized full Maxwell electrodynamic potential formulations using FIT. In: G. Manara (ed.) Proceedings of 2013 URSI International Symposium on Electromagnetic Theory (EMTS), pp. 1007-1010. IEEE (2013).

10. Baumanns, S., Selva Soto, M., Tischendorf, C.: Consistent initialization for coupled circuitdevice simulation. In: J. Roos, L.R.J. Costa (eds.) Scientific Computing in Electrical Engi- 
1 Systems of Differential Algebraic Equations in Computational Electromagnetics

neering SCEE 2008, Mathematics in Industry, vol. 14, pp. 297-304. Springer, Berlin (2010). DOI 10.1007/978-3-642-12294-1_38

11. Becks, T., Wolff, I.: Analysis of 3-d metallization structures by a full-wave spectral-domain technique. IEEE Trans. Microw. Theor. Tech. 40(12), 2219-2227 (1992). DOI 10.1109/22. 179883

12. Bedrosian, G.: A new method for coupling finite element field solutions with external circuits and kinematics. IEEE Trans. Magn. 29(2), 1664-1668 (1993). DOI 10.1109/20.250726

13. Boffi, D.: Finite element approximation of eigenvalue problems. Acta. Num. 19, 1-120 (2010). DOI 10.1017/S0962492910000012

14. Bondeson, A., Rylander, T., Ingelström, P.: Computational Electromagnetics. Texts in Applied Mathematics. Springer Berlin Heidelberg (2005). DOI 10.1007/b136922

15. Bossavit, A.: Whitney forms: a class of finite elements for three-dimensional computations in electromagnetism. IEE Proceedings 135(8), 493-500 (1988). DOI 10.1049/ip-a-1:19880077

16. Bossavit, A.: Differential geometry for the student of numerical methods in electromagnetism. Tech. rep., Électricité de France (1991).

17. Bossavit, A.: Computational Electromagnetism: Variational Formulations, Complementarity, Edge Elements. Academic Press, San Diego (1998).

18. Bossavit, A.: On the geometry of electromagnetism. (4): 'Maxwell's house'. JSAEM 6(4), 318-326 (1999)

19. Bossavit, A.: Stiff problems in eddy-current theory and the regularization of Maxwell's equations. IEEE Trans. Magn. 37(5), 3542-3545 (2001). DOI 00189464/01\$10.00

20. Bossavit, A., Kettunen, L.: Yee-like schemes on a tetrahedral mesh, with diagonal lumping. Int. J. Numer. Model. Electron. Network. Dev. Field 12(1-2), 129-142 (1999). DOI 10.1002/ (SICI)1099-1204(199901/04)12:1/2〈129::AID-JNM327〉3.0.CO;2-G

21. Bossavit, A., Kettunen, L.: Yee-like schemes on staggered cellular grids: a synthesis between FIT and FEM approaches. IEEE Trans. Magn. 36(4), 861-867 (2000). DOI 10.1109/20. 877580

22. Brenan, K.E., Campbell, S.L., Petzold, L.R.: Numerical solution of initial-value problems in differential-algebraic equations. SIAM, Philadelphia (1995)

23. Bíró, O., Preis, K.: On the use of the magnetic vector potential in the finite-element analysis of three-dimensional eddy currents. IEEE Trans. Magn. 25(4), 3145-3159 (1989). DOI $10.1109 / 20.34388$

24. Bíró, O., Preis, K.: Finite element analysis of 3-d eddy currents. IEEE Trans. Magn. 26(2), 418-423 (1990). DOI 10.1109/20.106343

25. Bíró, O., Preis, K., Richter, K.R.: Various FEM formulations for the calculation of transient 3d eddy currents in nonlinear media. IEEE Trans. Magn. 31(3), 1307-1312 (1995). DOI $10.1109 / 20.376269$

26. Carpenter, C.J.: Comparison of alternative formulations of 3-dimensional magnetic-field and eddy-current problems at power frequencies. IEE Proceedings B Electric Power Applications 127(5), 332 (1980). DOI 10.1049/ip-b:19800045

27. Chen, Q., Schoenmaker, W., Chen, G., Jiang, L., Wong, N.: A numerically efficient formulation for time-domain electromagnetic-semiconductor cosimulation for fast-transient systems. IEEE Trans. Comput. Aided. Des. Integrated Circ. Syst. 32(5), 802-806 (2013). DOI 10.1109/TCAD.2012.2232709

28. Clemens, M.: Large systems of equations in a discrete electromagnetism: formulations and numerical algorithms. IEE. Proc. Sci. Meas. Tech. 152(2), 50-72 (2005). DOI 10.1049/ ip-smt:20050849

29. Clemens, M., Schöps, S., De Gersem, H., Bartel, A.: Decomposition and regularization of nonlinear anisotropic curl-curl DAEs. COMPEL 30(6), 1701-1714 (2011). DOI 10.1108/ 03321641111168039

30. Clemens, M., Weiland, T.: Transient eddy-current calculation with the FI-method. IEEE Trans. Magn. 35(3), 1163-1166 (1999). DOI 10.1109/20.767155

31. Clemens, M., Weiland, T.: Regularization of eddy-current formulations using discrete graddiv operators. IEEE Trans. Magn. 38(2), 569-572 (2002). DOI 10.1109/20.996149 
32. Clemens, M., Wilke, M., Weiland, T.: Linear-implicit time-integration schemes for errorcontrolled transient nonlinear magnetic field simulations. IEEE Trans. Magn. 39(3), 11751178 (2003). DOI 10.1109/TMAG.2003.810221

33. Cortes Garcia, I., De Gersem, H., Schöps, S.: A structural analysis of field/circuit coupled problems based on a generalised circuit element. Submitted, 2018. arXiv: 1801.07081.

34. CST AG: CST STUDIO SUITE 2016 (2016). URL https: / / www . cst . com

35. De Gersem, H., Hameyer, K.: A finite element model for foil winding simulation. IEEE Trans. Magn. 37(5), 3472-3432 (2001). DOI 10.1109/20.952629

36. De Gersem, H., Hameyer, K., Weiland, T.: Field-circuit coupled models in electromagnetic simulation. J. Comput. Appl. Math. 168(1-2), 125-133 (2004). DOI 10.1016/j.cam.2003.05. 008

37. De Gersem, H., Weiland, T.: Field-circuit coupling for time-harmonic models discretized by the finite integration technique. IEEE Trans. Magn. 40(2), 1334-1337 (2004). DOI 10.1109/TMAG.2004.824536

38. Deschamps, G.A.: Electromagnetics and differential forms. Proc. IEEE 69(6), 676-696 (1981). DOI dx.doi.org/10.1109/PROC.1981.12048

39. Dirks, H.K.: Quasi-stationary fields for microelectronic applications. Electr. Eng. 79(2), 145-155 (1996). DOI 10.1007/BF01232924

40. Dutiné, J.S., Richter, C., Jörgens, C., Schöps, S., Clemens, M.: Explicit time integration techniques for electro- and magneto-quasistatic field simulations. In: R.D. Graglia (ed.) Proceedings of the International Conference on Electromagnetics in Advanced Applications (ICEAA) 2017. IEEE (2017). DOI 10.1109/ICEAA.2017.8065562

41. Dyck, D.N., Webb, J.P.: Solenoidal current flows for filamentary conductors. IEEE Trans. Magn. 40(2), 810-813 (2004). DOI 10.1109/TMAG.2004.824594

42. Eller, M., Reitzinger, S., Schöps, S., Zaglmayr, S.: A symmetric low-frequency stable broadband Maxwell formulation for industrial applications. SIAM J. Sci. Comput. 39(4), B703B731 (2017). DOI 10.1137/16M1077817

43. Estévez Schwarz, D.: Consistent initialization of differential-algebraic equations in circuit simulation. Tech. Rep. 99-5, Humboldt Universität Berlin, Berlin (1999)

44. Griffiths, D.F.: Introduction to Electrodynamics. Prentice-Hall, New Jersey (1999)

45. Gödel, N., Schomann, S., Warburton, T., Clemens, M.: GPU accelerated Adams-Bashforth multirate discontinuous Galerkin FEM simulation of high-frequency electromagnetic fields. IEEE Trans. Magn. 46(8), 2735-2738 (2010)

46. Hahne, P., Weiland, T.: $3 d$ eddy current computation in the frequency domain regarding the displacement current. IEEE Trans. Magn. 28(2), 1801-1804 (1992). DOI 10.1109/20.124056

47. Hairer, E., Nørsett, S.P., Wanner, G.: Solving Ordinary Differential Equations II: Stiff and Differential-Algebraic Problems, 2 edn. Springer Series in Computational Mathematics. Springer, Berlin (2002)

48. Harrington, R.F.: Field Computation by Moment Methods. Wiley-IEEE Press (1993)

49. Haus, H.A., Melcher, J.R.: Electromagnetic Fields and Energy. Prentice-Hall (1989).

50. Heaviside, O.: On the forces, stresses, and fluxes of energy in the electromagnetic field. Royal Society of London Proceedings Series I 50, 126-129 (1891)

51. Hehl, F.W., Obukhov, Y.N.: Foundations of Classical Electrodynamics - Charge, Flux, and Metric. Progress in Mathematical Physics. Birkhäuser, Basel (2003)

52. Heise, B.: Analysis of a fully discrete finite element method for a nonlinear magnetic field problem. SIAM J. Numer. Anal. 31(3), 745-759 (1994).

53. Hesthaven, J.S., Warburton, T.: Nodal discontinuous Galerkin methods: algorithms, analysis, and applications. Texts in applied mathematics. Springer, New York and Berlin (2008)

54. Jackson, J.D.: Classical Electrodynamics, 3rd edn. Wiley and Sons, New York (1998)

55. Jochum, M.T., Farle, O., Dyczij-Edlinger, R.: A new low-frequency stable potential formulation for the finite-element simulation of electromagnetic fields. IEEE Trans. Magn. 51(3), 7402,304 (2015). DOI 10.1109/TMAG.2014.2360080

56. Kameari, A.: Calculation of transient 3D eddy-current using edge elements. IEEE Trans. Magn. 26(5), 466-469 (1990). DOI 10.1109/20.106354 
57. Kerler-Back, J., Stykel, T.: Model reduction for linear and nonlinear magneto-quasistatic equations. Int. J. Numer. Meth. Eng. 111(13), 1274-1299 (2017). DOI 10.1002/nme.5507

58. Koch, S., Weiland, T.: Time domain methods for slowly varying fields. In: URSI International Symposium on Electromagnetic Theory (EMTS 2010), pp. 291-294 (2010). DOI 10.1109/ URSI-EMTS.2010.5636991

59. Koch, S., Weiland, T.: Different types of quasistationary formulations for time domain simulations. Radio Science 46(5) (2011). DOI 10.1029/2010RS004637

60. Lamour, R., März, R., Tischendorf, C.: Differential-Algebraic Equations: A Projector Based Analysis. Differential-Algebraic Equations Forum. Springer, Heidelberg (2013). DOI 10. 1007/978-3-642-27555-5

61. Larsson, J.: Electromagnetics from a quasistatic perspective. Am. J. Phys. 75(3), 230-239 (2007). DOI 10.1119/1.2397095

62. Manges, J.B., Cendes, Z.J.: Tree-cotree decompositions for first-order complete tangential vector finite elements. Int. J. Numer. Meth. Eng. 40(9), 1667-1685 (1997). DOI 10.1002/ (SICI)1097-0207(19970515)40:9〈1667::AID-NME133〉3.0.CO;2-9

63. Maxwell, J.C.: A dynamical theory of the electromagnetic field. Phil. Trans. R. Soc. London CLV, 459-512 (1864)

64. Mehrmann, V.: Index Concepts for Differential-Algebraic Equations, pp. 676-681. Springer Berlin Heidelberg, Berlin, Heidelberg (2015). DOI 10.1007/978-3-540-70529-1_120

65. Merkel, M., Niyonzima, I., Schöps, S.: Paraexp using leapfrog as integrator for highfrequency electromagnetic simulations. Radio Science 52(12), 1558-1569 (2017). DOI 10.1002/2017RS006357

66. Monk, P.: Finite Element Methods for Maxwell's Equations. Oxford University Press, Oxford (2003)

67. Monk, P., Süli, E.: A convergence analysis of Yee's scheme on nonuniform grids. SIAM J. Numer. Anal. 31(2), 393-412 (1994). DOI 10.1137/0731021

68. Munteanu, I.: Tree-cotree condensation properties. ICS Newsletter (International Compumag Society) 9, 10-14 (2002). URL http://www.compumag.org/jsite/images/ stories/newsletter/ICS-02-09-1-Munteanu.pdf

69. März, R.: Differential algebraic systems with properly stated leading term and MNA equations. In: K. Anstreich, R. Bulirsch, A. Gilg, P. Rentrop (eds.) Modelling, Simulation and Optimization of Integrated Circuits, pp. 135-151. Birkhäuser, Berlin (2003)

70. Nagel, L.W., Pederson, D.O.: Simulation program with integrated circuit emphasis. Tech. rep., University of California, Berkeley, Electronics Research Laboratory, ERL-M382 (1973)

71. Nicolet, A., Delincé, F.: Implicit Runge-Kutta methods for transient magnetic field computation. IEEE Trans. Magn. 32(3), 1405-1408 (1996). DOI 0.1109/20.497510

72. Nédélec, J.C.: Mixed finite elements in $r^{3}$. Numerische Mathematik 35(3), 315-341 (1980). DOI 10.1007/BF01396415

73. Ostrowski, J., Hiptmair, R., Krämer, F., Smajic, J., Steinmetz, T.: Transient full Maxwell computation of slow processes. In: B. Michielsen, J.R. Poirier (eds.) Scientific Computing in Electrical Engineering SCEE 2010, Mathematics in Industry, vol. 16, pp. 87-95. Springer, Berlin (2012). DOI 10.1007/978-3-642-22453-9_10

74. Ouédraogo, Y., Gjonaj, E., Weiland, T., De Gersem, H., Steinhausen, C., Lamanna, G., Weigand, B., Preusche, A., Dreizler, A., Schremb, M.: Electrohydrodynamic simulation of electrically controlled droplet generation. International Journal of Heat and Fluid Flow 64, 120-128 (2017)

75. Petzold, L.R.: Differential/algebraic equations are not ODE's. SIAM J. Sci. Stat. Comput. 3(3), 367-384 (1982). DOI 10.1137/0903023

76. Rapetti, F., Rousseaux, G.: On quasi-static models hidden in Maxwell's equations. APNUM 79, 92-106 (2014). DOI 10.1016/j.apnum.2012.11.007

77. Rautio, J.C.: The long road to Maxwell's equations. IEEE Spectrum 51(12), 36-56 (2014). DOI 10.1109/MSPEC.2014.6964925

78. Raviart, P.A., Thomas, J.M.: Primal hybrid finite element methods for 2nd order elliptic equations. Math. Comput. 31(138), 391-413 (1977) 
79. Ruehli, A.E.: Equivalent circuit models for three-dimensional multiconductor systems. IEEE Trans. Microw. Theor. Tech. 22(3), 216-221 (1974)

80. Ruehli, A.E., Antonini, G., Jiang, L.: The Partial Element Equivalent Circuit Method for Electro-Magnetic and Circuit Problems. Wiley and Sons, Hoboken, New Jersey (2015)

81. Schilders, W.H.A., Ciarlet, P., ter Maten, E.J.W. (eds.): Handbook of Numerical Analysis. Numerical Methods in Electromagnetics, Handbook of Numerical Analysis, vol. 13. NorthHolland (2005)

82. Schmidt, K., Sterz, O., Hiptmair, R.: Estimating the eddy-current modeling error. IEEE Trans. Magn. 44(6), 686-689 (2008). DOI 10.1109/TMAG.2008.915834

83. Schoenmaker, W.: Computational Electrodynamics. River Publishers Series in Electronic Materials and Devices. River Publishers (2017)

84. Schuhmacher, S., Klaedtke, A., Keller, C., Ackermann, W., De Gersem, H.: Optimizing the inductance cancellation behavior in an EMI filter design with the help of a sensitivity analysis. In: EMC Europe. Angers, France (2017)

85. Schuhmann, R., Weiland, T.: Conservation of discrete energy and related laws in the finite integration technique. PIER 32, 301-316 (2001). DOI 10.2528/PIER00080112.

86. Schöps, S.: Multiscale modeling and multirate time-integration of field/circuit coupled problems. Dissertation, Bergische Universität Wuppertal \& Katholieke Universiteit Leuven, Düsseldorf (2011). VDI Verlag. Fortschritt-Berichte VDI, Reihe 21

87. Schöps, S., Bartel, A., Clemens, M.: Higher order half-explicit time integration of eddy current problems using domain substructuring. IEEE Trans. Magn. 48(2), 623-626 (2012). DOI 10.1109/TMAG.2011.2172780

88. Schöps, S., De Gersem, H., Weiland, T.: Winding functions in transient magnetoquasistatic field-circuit coupled simulations. COMPEL 32(6), 2063-2083 (2013). DOI 10.1108/COMPEL-01-2013-0004

89. Steinmetz, T., Kurz, S., Clemens, M.: Domains of validity of quasistatic and quasistationary field approximations. COMPEL 30(4), 1237-1247 (2011). DOI 10.1108/ 03321641111133154

90. Taflove, A.: Computational Electrodynamics: The Finite-Difference Time-Domain-Method. Artech House, Dedham, MA (1995)

91. Taflove, A.: A perspective on the 40-year history of FDTD computational electrodynamics. Appl. Comput. Electromagn. 22(1), 1-21 (2007)

92. Tischendorf, C.: Topological index calculation of DAEs in circuit simulation. Tech. Rep. 3-4, Humboldt Universität Berlin, Berlin (1999)

93. Tonti, E.: On the formal structure of physical theories. Tech. rep., Politecnico di Milano, Milano, Italy (1975)

94. Tsukerman, I.A.: Finite element differential-algebraic systems for eddy current problems. Numer. Algorithm. 31(1), 319-335 (2002). DOI 10.1023/A:1021112107163

95. Webb, J.P., Forghani, B.: The low-frequency performance of $h-\phi$ and $t-\omega$ methods using edge elements for 3d eddy current problems. IEEE Trans. Magn. 29(6), 2461-2463 (1993). DOI 10.1109/20.280983

96. Weeks, W., Jimenez, A., Mahoney, G., Mehta, D., Qassemzadeh, H., Scott, T.: Algorithms for ASTAP - a network-analysis program. IEEE Trans. Circ. Theor. 20(6), 628-634 (1973). DOI 10.1109/TCT.1973.1083755

97. Weiland, T.: A discretization method for the solution of Maxwell's equations for sixcomponent fields. Int. J. Electron. Commun. (AEU) 31, 116-120 (1977)

98. Weiland, T.: On the unique numerical solution of Maxwellian eigenvalue problems in three dimensions. Particle Accelerators 17(227-242) (1985)

99. Weiland, T.: Time domain electromagnetic field computation with finite difference methods. Int. J. Numer. Model. Electron. Network. Dev. Field 9(4), 295-319 (1996). DOI 10.1002/ (SICI)1099-1204(199607)9:4〈295::AID-JNM240〉3.0.CO;2-8

100. Yee, K.S.: Numerical solution of initial boundary value problems involving Maxwell's equations in isotropic media. IEEE Trans. Antenn. Propag. 14(3), 302-307 (1966). DOI 10.1109/TAP.1966.1138693 